\title{
Carbon Nanotube Spaced Graphene Aerogels with Enhanced Capacitance in Aqueous and Ionic liquid Electrolytes
}

\author{
Qingguo Shao, ${ }^{\mathrm{ab}}$ Jie Tang, ${ }^{\mathrm{ab}}$ Yuexian Lin, ${ }^{\mathrm{ab}}$ Jing Li ${ }^{\mathrm{ab}}$ Faxiang Qin, ${ }^{\mathrm{a}}$ Jinshi Yuan, \\ and Lu-Chang Qin ${ }^{\mathrm{c}}$ \\ ${ }^{a}$ National Institute for Materials Science, 1-2-1 Sengen, Tsukuba 305-0047, Japan. E-mail: \\ tang.jie@nims.go.jp \\ ${ }^{b}$ Doctoral Program in Materials Science and Engineering, University of Tsukuba, 1-1-1 Tennodai, \\ Tsukuba 305-8577, Japan. E-mail:tang.jie@nims.go.jp \\ ${ }^{c}$ Department of Physics and Astronomy, University of North Carolina at Chapel Hill, NC \\ 27599-3255,USA.E-mail:lcqin@unc.edu
}

\begin{abstract}
Carbon nanotube spaced graphene aerogel has been prepared by a hydrothermal method and used for supercapacitor applications. The specific surface area and specific capacitance can be controlled by tuning the amount of added carbon nanotubes. The as-prepared composite aerogel keeps the advantage of aerogel structure in providing macropores to ensure electrodes fast wetted by the electrolyte ions, and also possesses additional mesopores created by the carbon nanotube spacers for more ion adsorption. Benefited from that, the composite aerogel exhibit significantly enhanced supercapacitor properties in both aqueous and ionic liquid electrolyte. Compared with graphene aerogels, the composite aerogels show a $37 \%$
\end{abstract}


larger specific capacitance of $245.5 \mathrm{~F} \mathrm{~g}^{-1}$ at a current density of $2.5 \mathrm{~A} \mathrm{~g}^{-1}$, and high rate capability of $197.0 \mathrm{~F} \mathrm{~g}^{-1}$ at a high current density of $80 \mathrm{~A} \mathrm{~g}^{-1}$ in aqueous electrolyte. Moreover, the composite aerogels also deliver a $33 \%$ larger specific capacitance of $183.3 \mathrm{~F} \mathrm{~g}^{-1}$ at $0.5 \mathrm{~A} \mathrm{~g}^{-1}$ and a high energy density of $80 \mathrm{Wh} \mathrm{kg}^{-1}$ when using an ionic liquid $\left(\mathrm{EMIMBF}_{4}\right)$ as the electrolyte.

Key words: Graphene, Carbon nanotube, Aerogel, Supercapacitor, Ionic liquid

* Corresponding author: Jie Tang

Email: tang.jie@nims.go.jp 


\section{Introduction:}

Electric double-layer capacitors (EDLC), also often known as supercapacitors, have attracted great attention in recent years due to their high power density and long cycle life. ${ }^{1-3}$ Among of the various materials for supercapacitors, graphene has appeared to be a promising candidate because of its large specific surface area, high electrical conductivity, and outstanding mechanical properties. ${ }^{4-6}$ However, the graphene sheets tend to restack due to the $\pi-\pi$ interactions and the van der Waals forces between them, which make it often difficult for ions to gain access to the inner layers of stacked graphene to form electric double-layers with the electrolyte, thus usually leading to an actual specific capacitance far below its theoretical value of 550 $\mathrm{F} \mathrm{g}^{-1}$ calculated for graphene. ${ }^{7}$ To tackle this problem, many efforts have been devoted to synthesis of graphene materials with a desired structure to reduce the restacking of graphene, such as having curved graphene sheets, folded graphene paper, nano-porous graphene foam, vertically aligned graphene sheets, and our recently reported graphene hollow spheres. ${ }^{8-12}$

Recently, graphene aerogel (GA), a three-dimensional (3D) macroscopic architecture of graphene, has been found to exhibit great potential in the fields of oil adsorption and catalysis. Based on their outstanding properties such as large specific surface area, high porosity, and high electrical conductivity, GAs have also been used as the electrode material of supercapacitors. Lee et al. reported a ball-shaped graphene aerogel produced by a chemical vapor deposition (CVD) method, which showed a

specific capacitance of $206 \mathrm{~F} \mathrm{~g} \mathrm{~g}^{-1} \cdot{ }^{13}$ Despite the high purity and superior electrical 
conductivity of the CVD produced graphene aerogel, the low yield and required high operating temperature condition hindered its practical applications. $\mathrm{Xu}$ et al. developed a hydrothermal method to produce graphene hydrogels. ${ }^{14}$ The as-prepared graphene hydrogel is electrically conductive, mechanically strong, thermally stable, and exhibited a specific capacitance of $160 \mathrm{~F} \mathrm{~g}^{-1} \cdot{ }^{14}$ On the other hand, by freeze-drying the hydrothermally produced graphene based hydrogels, various graphene aerogels including $\mathrm{N}$-doped graphene aerogels, $\mathrm{N}$ and $\mathrm{B}$ co-doped graphene aerogels, graphene/porous carbon aerogels, and graphene/polypyrrole aerogels have also been prepared as the electrode materials for supercapacitors. ${ }^{14-16}$ There is a distinct advantage for these graphene based aerogels for supercapacitors that the graphene based aerogels usually possess a high volume of large pores (>100 nm), which would allow the outermost graphene sheets to be quickly wetted by the electrolyte. As a result, the assembled supercapacitors could be charged and discharged very quickly. However, there also exists a main drawback. Graphene sheets in these graphene aerogels usually stacked to form a thick slab between the macopores, leaving behind the inner layers inaccessible to the electrolyte ions, which makes the current graphene aerogel based electrodes suffer from a low specific capacitance.

To prevent the restacking of graphene, our group has reported that single-walled carbon nanotubes (SWCNTs) are an effective spacer to inhibit the restacking of chemically reduced graphene. ${ }^{19,20}$ Subsequently, carbon black, small graphite particles, and diethylene glycol have also been studied recently as 
spacers. $^{21-23}$ Zhu et al. reported that carbon nanotubes were also good spacers for CVD grown graphene and the supercapacitor showed a high energy density of $60 \mathrm{Wh}$ $\mathrm{kg}^{-1}$ in ionic liquid electrolyte. ${ }^{24}$ More recently, we have developed a hydrothermally reduced carbon nanotube/graphene composite for selective detection of uric acid in which the carbon nanotubes played a key role in increasing the active sites for oxidation of uric acid. ${ }^{25}$ These works led us to use the carbon nanotube spacer to prevent the restacking in graphene aerogels, which would result in a macro-porous aerogel structure with separated graphene walls to provide both macropores to ensure electrodes fast wetted by the electrolyte ions and additional mesopores induced by the carbon nanotube spacers for more ion adsorption.

Herewith, we report a comprehensive study of the SWCNT spaced graphene aerogel (SSGA) materials for supercapacitor applications and investigate the role of the SWCNT spacer in realizing a high specific capacitance together with a high rate capability by increasing the specific surface area, bringing about favorable pore size distribution and resulting in faster ion transport. Compared with previously reported graphene aerogel electrodes for supercapacitors, the combined effects of the macro-porous structure and the SWCNT spacer in the composite aerogel are : (i) The three-dimensional (3D) macro-porous structure provides numerous large pores to make the electrode be wetted quickly by the electrolyte to ensure high rate performance; (ii) The SWCNTs are in between the graphene layers to prevent the restacking of graphene sheets and more accessible surface area is made available for ion adsorption; (iii) The introduction of highly conductive SWCNTs can also reduce 
the electrical resistance of the SSGA electrode to further increase the rate capability and promote stable cycling performance.

Electrochemical tests showed that the SSGA-12 sample (weight ratio of graphene oxide to SWCNT is 12:1 in the initial composite) delivered a highest specific capacitance of $245.5 \mathrm{~F} \mathrm{~g}^{-1}$ at a current density of $2.5 \mathrm{~A} \mathrm{~g}^{-1}$ in aqueous electrolyte, which is $37 \%$ higher than that of pristine graphene aerogel (GA). Besides, this value is also much higher than that of previously reported 3D macroscopic graphene hydrogels ( $\left.160 \mathrm{~F} \mathrm{~g}^{-1}, 166 \mathrm{~F} \mathrm{~g}^{-1}, 186 \mathrm{~F} \mathrm{~g}^{-1}, 190.1 \mathrm{~F} \mathrm{~g}^{-1}\right),{ }^{14,26-28}$ graphene aerogels $\left(128 \mathrm{~F} \mathrm{~g}^{-1}, 239 \mathrm{~F} \mathrm{~g}^{-1}\right),{ }^{16,29}$ folded graphene paper $\left(172 \mathrm{~F} \mathrm{~g}^{-1}\right){ }^{8}$ or graphene foams $\left(110 \mathrm{~F} \mathrm{~g}^{-1}\right) .{ }^{9} \mathrm{Using}$ this structure the rate capability has also been improved significantly. A high specific capacitance of $197.0 \mathrm{~F} \mathrm{~g}^{-1}$ is achieved even at a high current density of $80 \mathrm{~A} \mathrm{~g}^{-1}$, corresponding to $80 \%$ of that at $2.5 \mathrm{~A} \mathrm{~g}^{-1}$. After 2000 cycles of repeated charging and discharging at a current density of $10 \mathrm{~A} \mathrm{~g}^{-1}, 97 \%$ of its initial capacitance is retained, highlighting its excellent stability. When using the ionic liquid (EMIMBF 4$)$ electrolyte, the SSGA-12 electrode exhibited a capacitance of $183.3 \mathrm{~F} \mathrm{~g}^{-1}$ at $0.5 \mathrm{~A} \mathrm{~g}^{-1}$ and a high energy density of $80 \mathrm{Wh} \mathrm{kg}^{-1}$, which is about an order of magnitude larger than the previously reported 3D macroscopic graphene electrodes in aqueous electrolyte.

\section{Experimental}

\subsection{Sample Preparation}


Graphene oxide (GO) was obtained first from natural graphite flakes by a modified Hummer's method. ${ }^{30}$ The SWCNT/graphene composite aerogel was synthesized by hydrothermal reduction of a mixture of the graphite oxide and SWCNTs, followed by freeze drying. In our experiment the graphene oxide dispersion was mixed with carbon nanotubes with different weight ratios of GO to SWCNTs $(16: 1,12: 1$ and 8:1). The mixture was then sonicated for 30 minutes before it was transferred to a Teflon lined autoclave and held in an oven at $180{ }^{\circ} \mathrm{C}$ for 12 hours. After being cooled to room temperature, the obtained composite hydrogel was freeze dried to obtain an aerogel. The products were denoted as SSGA-16, SSGA-12, SSGA-8 according to the weight ratio of GO to SWCNTs in the starting mixture. For comparison pristine graphene aerogel (GA) was also prepared through a similar procedure without adding carbon nanotubes.

\subsection{Structural Characterization}

The morphology and microstructure of the as-prepared material was examined with both scanning electron microscopy (SEM, JEOL 6500F) and transmission electron microscopy (TEM, JEOL 2100). The nitrogen adsorption/desorption isotherms at $77 \mathrm{~K}$ were collected on an AUTOSORB iQ-MP instrument at $77 \mathrm{~K}$. The specific surface area was determined according to the Brunauer-Emmett-Teller (BET) method in the relative pressure range of $0.05-0.3$. The pore size distribution was analyzed using a BJH model from the nitrogen adsorption data. X-ray diffraction (XRD) patterns were obtained with a Rigaku RINT 2500 diffractometer. Fourier transform infrared spectroscopy (FT-IR) data were acquired on a Nicolet 6700 
spectrometer and Raman spectra were obtained using RAMAN-11 (Nanophoton) with a $532 \mathrm{~nm}$ laser.

\subsection{Electrochemical Characterization}

The graphene aerogels were used as the active material for the electrodes of symmetrical supercapacitors for electrochemical characterization. To assemble a test cell, the as-prepared aerogels were cut into small slices with a thickness of about 1 $\mathrm{mm}$ for preparation of electrodes. For tests in aqueous electrolyte, $5 \mathrm{M} \mathrm{KOH}$ was used as the electrolyte, a piece of PTFE filter paper was used as the separator, and two Pt foils were used as the current collectors. For tests in ionic liquid electrolyte, 1-Ethyl-3-methylimidazolium tetrafluoroborate $\left(\mathrm{EMIMBF}_{4}\right)$ was used as the electrolyte, a piece of glass fiber was used as the separator, and conductive carbon coated aluminum foils (Exopack ${ }^{\mathrm{TM}} 0.5$ mil 2-side coating) were used as the current collectors. The cells were assembled in an argon filled glove box.

Cyclic voltammetry (CV), galvanostatic charge-discharge (GC), and electrical impedance spectroscopy (EIS) measurements were performed using a VMP3 multi-potentiostat/galvanostat (Biologic) system. The specific capacitance $C$ was calculated by $C=2 I \Delta t /(m \Delta V)$, where $I$ is the discharge current, $\Delta t$ is the discharge time, $\Delta V$ is the voltage change excluding the IR drop during the discharge process, and $m$ is the mass of a single electrode. The specific energy density $E$ and the specific power density $P$ were calculated using $E=C V^{2} / 8$ and $P=E / \Delta t$, respectively, where $C$ is the specific capacitance, $V$ is the voltage of the charged supercapacitor, and $\Delta t$ is the discharge time. 


\section{Results and Discussion}

\subsection{Preparation and Morphology}

Figure 1(a) illustrates the synthesis of single-walled carbon nanotube spaced graphene aerogel (SSGA). The monitoring photograph in each step is shown in Fig. 1(b). As illustrated in Fig. 1(a), the SWCNTs were first dispersed in a solution of GO. Considering that the aqueous solution of GO was a good solvent for dispersing SWCNTs and the single layer nature of the GO sheets, ${ }^{31,32}$ the mixture of SWCNTs and single layers of GO is homogeneous as seen in Fig. 1(b). During the process of hydrothermal reduction, due to the increasing hydrophobicity of the reduced GO caused by losing the oxygenous groups, the hydrothermally reduced GO sheets would self-assemble into a 3D macro-porous structure with carbon nanotubes between them, forming a SWCNT/graphene composite hydrogel. Finally, the composite hydrogel was freeze dried to obtain a composite aerogel. It was noticed that the composite aerogel is ultralight in weight. As shown in Fig. 1(b), a block of the composite aerogel could be balanced on a dandelion, indicating its ultralight weight due to its porous structure which will be discussed later.

The unique structure of the SSGAs was characterized by SEM and TEM as shown in Fig. 2. As can be seen in the SEM images given in Fig. 2(a) and 2(b), the graphene aerogels have a 3D macro-porous structure with macropores $(5-10 \mu \mathrm{m})$ and graphene walls. In addition, the TEM image shown in Fig. 2(c) reveals that the carbon nanotubes were randomly mixed with graphene sheets, indicating that the carbon 
nanotubes and graphene sheets are well mixed. Further evidence is shown in the high-resolution TEM image given in Fig. 2(d) which shows a single carbon nanotube sticking out of a graphene sheet, illustrating that the SWCNTs are in between the graphene layers, forming a sandwich structure. Combining the SEM and TEM images, we suggest that the SSGAs possess multi-level of pores and the graphene sheets within the structure are well spaced by carbon nanotubes. These features will not only guarantee that the SSGA electrode can be wetted quickly by the electrolyte but also help to release more accessible surface area for electrolyte ion adsorption in the supercapacitor device.

\subsection{Structural Study}

The structure of GA and SSGAs were further investigated by XRD, FT-IR spectroscopy, and Raman spectroscopy. Fig. 3(a) displays the XRD patterns of GO, GA, and SSGA. The characteristic inter-layer spacing of the GO layers as indicated by the sharp reflection peak at $2 \theta=11.4^{\circ}$ is $d=0.776 \mathrm{~nm}$, which is much larger than that of graphite $(\sim 0.340 \mathrm{~nm})$ due to the introduction of oxygenous functional groups. After the hydrothermal reduction this characteristic peak vanished and a new and broad peak centered at about $2 \theta=23.7^{\circ}$ (corresponding to $d=0.375 \mathrm{~nm}$ ) was observed in both GA and SSGA, reflecting the deoxygenization and partial recovery of the graphitic structure. It should also be noted that the weaker and broader peak of SSGA (in comparison with that of GA) indicates that a lower degree of restacking of graphene in SSGA, which is attributed to the SWCNT spacers. In the FT-IR spectra 
(Fig. 3(b)) the $\mathrm{C}=\mathrm{O}$ peak in $\mathrm{GO}$ at $1730 \mathrm{~cm}^{-1}$ disappeared in GA and SSGA, confirming the reduction of GO. Fig. 3(c) shows the Raman spectra of GA, SSGA, and SWCNTs collected in the window of 600 and $1200 \mathrm{~cm}^{-1}$. The intensity ratio of the $\mathrm{D}$ band at $1335 \mathrm{~cm}^{-1}$ to the $\mathrm{G}$ band at $1578 \mathrm{~cm}^{-1}$ of SSGAs decreased gradually from 1.00 to 0.72 with the increasing concentration of SWCNTs in the composite, indicating successive increment of perfect graphitic structures due to SWCNTs.

It is well known that the supercapacitor performance is highly sensitive to the specific surface area (SSA) and the pore size distribution of the carbon electrode. Previous reports have shown that a large SSA together with a suitable pore size distribution containing a large proportion of accessible mesopores is ideal for electrolyte ion adsorption. ${ }^{33}$ The porosity of the as-prepared GA and SSGA was studied by nitrogen adsorption/desorption isotherms. As shown in Fig. 4(a), the isotherm of GA displays a steep uptake at the relative pressure of 0.9-1.0, attributed to the large number of textural macropores. As for the SSGA, the curves are the typical type IV isotherms, indicating a large proportion of mesopores. The difference in the pore size distribution between GA and SSGA is further revealed in the plot of cumulative pore volume versus pore diameter shown in Fig. 4(b). Pores smaller than $20 \mathrm{~nm}$ accounted for only $44 \%$ of the total volume in GA, while nearly $90 \%$ of the total pore volume is contributed by pores smaller than $20 \mathrm{~nm}$ in SSGA, indicating a large number of smaller mesopores have been created by the insertion of SWCNTs. The calculated SSAs of the four samples are given in Table 1. All the three SSGA 
samples possess larger SSA than GA and the SSGA-12 sample showed the largest SSA of $807 \mathrm{~m}^{2} \mathrm{~g}^{-1}$, which is even comparable to activated graphene aerogel or holey graphene. ${ }^{34,35}$ Therefore, the incorporation of SWCNTs not only has changed the pore size distribution but has also increased the SSA, which is helpful for more ion adsorption. These changes in pore size and SSA can be understood in terms of the unique structure of the SSGA as shown in Fig. 2. The SWCNTs are sandwiched between the graphene layers to serve as spacers and, as a result, more graphene sheets are separated, which would in turn produce more mesopores and larger SSA. It should also be noted that excessive amount of carbon nanotubes in the composite would actually decrease the SSA (the SSA of SSGA-8 is lower than SSGA-12), which is ascribed to the lower SSA $\left(344 \mathrm{~m}^{2} \mathrm{~g}^{-1}\right)$ of SWCNTs than that of graphene.

\subsection{Capacitance in Aqueous Electrolyte}

The supercapacitor performance using the aerogel as the electrodes was first investigated in aqueous electrolyte $(5 \mathrm{M} \mathrm{KOH})$. The cyclic voltammograms $(\mathrm{CVs})$ of GA, SSGA-16, SSGA-12, and SSGA-8 at different scan rates are shown in Fig. 5(a-d). It is observed that all CVs featured the rectangular loops, indicating that the charges stored in the electrodes are dominated by the electric double-layer mechanism. Besides, all the CV loops retained the rectangular geometry even at a high scan rate of $500 \mathrm{mV} \mathrm{s}^{-1}$, suggesting their excellent rate capability, which is ascribed to the vast macropores in the aerogel that allow the electrode to be fast wetted by the electrolyte. To make a close comparison among the four electrodes, the CVs of different 
electrodes at $100 \mathrm{mV} \mathrm{s}^{-1}$ are plotted in Fig. 5(e). The area of the $\mathrm{CV}$ loop is proportional to the charge storage capacity. As can be seen in the figure, all the three SSGA samples showed larger CV areas than the GA sample, indicating improved capacitance after incorporating the SWCNTs. The specific capacitance calculated on the scan rate is shown in Fig. 5(f). SSGA-12 exhibited the largest capacitance of $246.9 \mathrm{~F} \mathrm{~g}^{-1}$ at $50 \mathrm{mV} \mathrm{s}^{-1}$, which decreased to $206.9 \mathrm{~F} \mathrm{~g}^{-1}$ (16\% decrease) when the scan rate increased to $500 \mathrm{mV} \mathrm{s}^{-1}$. In contrast, GA showed a much lower capacitance of $179.3 \mathrm{~F} \mathrm{~g}^{-1}$ at $50 \mathrm{mV} \mathrm{s}^{-1}$ and a larger decrease of $24 \%$ to $135.9 \mathrm{~F} \mathrm{~g}^{-1}$ at $500 \mathrm{mV} \mathrm{s}^{-1}$. The CV results indicate that both the specific capacitance and the rate capability are improved significantly by introducing SWCNTs into the graphene aerogel.

Galvanostatic charge/discharge tests were performed to further investigate the supercapacitor properties of the aerogel electrodes in aqueous electrolyte. Fig. 6(a-d) are the charge/discharge profiles of GA, SSGA-16, SSGA-12, and SSGA-8 at different current densities from 5 to $80 \mathrm{~A} \mathrm{~g}^{-1}$. As can be observed, all the curves are linear and symmetric and all have a negligible IR drop, which further confirms the ideal electric double-layer capacitance. For comparison, the charge/discharge curves of different samples at a constant current density of $5 \mathrm{~A} \mathrm{~g}^{-1}$ are shown in Fig. 6(e). Evidently, the charge and discharge time of all the three SSGA samples are longer than the GA sample at the same current density, indicating that the SSGA electrodes can store more energy than the GA electrode. The calculated specific capacitance of the aerogels under different current densities is presented in Fig. 6(f). The specific 
capacitance at a loading current density of $2.5 \mathrm{~A} \mathrm{~g}^{-1}$ for GA, SSGA-16, SSGA-12 and SSGA-8 are $179.0,206.0,245.5$, and $230.5 \mathrm{~F} \mathrm{~g}^{-1}$, respectively. The specific capacitance is very close to the value calculated from the CVs and also exhibited the same trend, indicating their high rate capability. Compared with GA, all the SSGA samples exhibited improved capacitance. In addition, it is interesting to note that the specific capacitance of the four samples are all in accordance with their BET SSA listed in Table 1, which further confirms the usefulness and functionalities of having SWCNT spacers to realize more accessible surface area and favorable pore structures. In particular the SSGA-12 sample shows the largest increment of 37\% to reach 245.5 $\mathrm{F} \mathrm{g}^{-1}$, which is much higher than that of previously reported 3D macroscopic graphene based electrodes in aqueous electrolyte, i.e., graphene hydrogels $\left(160 \mathrm{~F} \mathrm{~g} \mathrm{~g}^{-1}, 166 \mathrm{~F} \mathrm{~g}^{-1}\right.$, $\left.186 \mathrm{~F} \mathrm{~g}^{-1}, 190.1 \mathrm{~F} \mathrm{~g}^{-1}\right),{ }^{14,26-28}$ graphene aerogels $\left(128 \mathrm{~F} \mathrm{~g}^{-1}, 239 \mathrm{~F} \mathrm{~g}^{-1}\right),{ }^{16,29}$ folded graphene paper $\left(172 \mathrm{~F} \mathrm{~g} \mathrm{~g}^{-1}\right),{ }^{8}$ or graphene foams $\left(110 \mathrm{~F} \mathrm{~g}^{-1}\right) .{ }^{9}$ Furthermore, when the current density is increased from 2.5 to $80 \mathrm{~A} \mathrm{~g}^{-1}$, the specific capacitance of GA, SSGA-16, SSGA-12, and SSGA-8 becomes 121.0, 159.1, 197.0, and $192.3 \mathrm{~F} \mathrm{~g}^{-1}$, corresponding to a retention of $68 \%, 77 \%, 80 \%$, and $83 \%$, respectively. It is clear that the rate capability is also improved after incorporating highly conductive SWCNTs to the graphene aerogel electrode.

\subsection{Capacitance in Ionic liquid Electrolyte}

To explore further the electrochemical performance of the prepared graphene 
aerogels, $\mathrm{EMIMBF}_{4}$, a room temperature ionic liquid with a wide potential window was utilized as the electrolyte and the graphene aerogel electrodes were characterized in the voltage range of 0 to $3.5 \mathrm{~V}$. Fig. 7(a) compares the CVs of GA and SSGAs at the scan rate of $50 \mathrm{mV} \mathrm{s}^{-1}$. Just the same as in aqueous electrolyte, rectangular CVs were obtained, indicating the ideal electric double-layer capacitance in the ionic liquid electrolyte and SSGA-12 again showed the largest specific capacitance. Fig. 7(b) shows the CV of SSGA-12 at different scan rates from 20 to $100 \mathrm{mV} \mathrm{s}^{-1}$. Figure 6(c) and 6(d) charge/discharge curves at current density of 1 and $5 \mathrm{~A} \mathrm{~g}^{-1}$, respectively. Figure 7(e) shows the charge/discharge curves of SSGA-12 at different current densities from 1 to $10 \mathrm{~A} \mathrm{~g}^{-1}$. As observed in the plots, the $\mathrm{CV}$ s retained the rectangular shape even at a high scan rate of $100 \mathrm{mV} \mathrm{s}^{-1}$, and the charge/discharge curves followed the geometry of an isosceles triangle even at $10 \mathrm{~A} \mathrm{~g}^{-1}$, indicating its excellent stability in the ionic liquid electrolyte. Fig. 7(f) displays the calculated specific capacitance of the aerogels under different current densities. At a low current density of $0.5 \mathrm{~A} \mathrm{~g} \mathrm{~g}^{-1}$, the specific capacitance of GA, SSGA-16, SSGA-12, and SSGA -8 are $137.4,157.0,183.3$, and $167.9 \mathrm{~F} \mathrm{~g}^{-1}$, respectively. All the capacitance values are understandably lower than that in the aqueous electrolyte due to the fact that the ionic liquid has larger molecular ions and larger viscosity. Compared with GA, all the SSGA samples also showed improved capacitance in the ionic liquid electrolyte, and the SSGA-12 sample showed the largest increment of $33 \%$ to a high capacitance of 183.3 $\mathrm{F} \mathrm{g}^{-1}$. Moreover, when the current density is increased by 20 times to $10 \mathrm{~A} \mathrm{~g}^{-1}$, the specific capacitance for GA is only $97 \mathrm{~F} \mathrm{~g}^{-1}$. However, thanks to the SWCNT 
spacers, a much higher capacitance, $154.3 \mathrm{~F} \mathrm{~g}^{-1}$, which is $60 \%$ larger than that of GA, was retained for SSGA-12. Such an excellent rate performance demonstrates the rapid charge/discharge capability of the SSGA-12 electrode, which is particularly important and useful for supercapacitors requiring high energy densities.

\subsection{EIS Analysis}

Electrochemical impedance spectroscopy (EIS) was also carried out to understand the mechanism responsible for the improved supercapacitor performance after having additive SWCNTs in the graphene aerogels. Fig. 8(a) and 8(b) show the Nyquist plots of the EIS data from GA and various SSGA electrodes in aqueous electrolyte and ionic liquid electrolyte, respectively. As can be observed, each plot consists of a semicircle at high frequencies and an almost vertical line at low frequencies, where the behaviors become mainly capacitive. The intercept on the horizontal axis gives the equivalent series resistance (ESR). The ESR of the SSGA samples gradually decreased with increasing concentration of SWCNTs, attributed to the higher conductivity of SWCNTs. The semicircle in the Nyquist plot is closely related to the transport of electrolyte ions at the electrode/electrolyte interface and the radius of the semicircle is indicative of the charge transfer resistance $\left(R_{c t}\right)$, which is associated with the porous structure of the electrode. ${ }^{36}$ Generally, the smaller the radius of the semicircle, the higher the ion transport ability into the pores of the sample. $^{37-39}$

Table 2 lists the ESR and $\mathrm{R}_{\mathrm{ct}}$ of the tested graphene aerogel samples. In aqueous electrolyte, the $\mathrm{R}_{\mathrm{ct}}$ values of SSGAs are approximately only one half of GA 
and also decreased with increasing concentration of SWCNTs. This result means that the electrolyte ions could diffuse more easily in the SSGA samples, which is attributed to that the added SWCNTs served as spacers to increase the inter-layer spacing of graphene sheets and to inhibit their restacking. The $\mathrm{R}_{\mathrm{ct}}$ values obtained in the ionic liquid electrolyte also showed the same trend. As can be observed, the values of ESR and $\mathrm{R}_{\mathrm{ct}}$ of each sample are both larger than that in the aqueous electrolyte due to the lower conductivity and much higher viscosity of the ionic liquid electrolyte. The $\mathrm{R}_{\mathrm{ct}}$ of GA, SSGA-16, SSGA-12, and SSGA-8 are 18.5, 11.5, 5.0, and $4.3 \Omega$, respectively. The reduced $\mathrm{R}_{\mathrm{ct}}$ after incorporating SWCNTs means that the diffusion of ions in the SSGA samples is more easily, which confirms again that the SWCNT spacers played an effective role in preventing the restacking of graphene sheets, leading to improved capacitance and rate capability. We also noted that the $\mathrm{R}_{\mathrm{ct}}$ of SSGA-8 is almost the same as that of SSGA-12, indicating that further increases of SWCNTs in the composite electrode will not reduce it further. Instead, it will actually add more weight to the electrode and thus the optimal ratio of SWCNTs to GO is 1:12, which is in agreement with the electrochemical results.

\subsection{Ragone Plot and Cycle S1tability}

The energy density of a supercapacitor is a vital indicator for its quality. The Ragone plot of the SSGA-12 supercapacitor showing the relationship between the specific energy density $(E)$ and the specific power density $(P)$ is displayed in Fig. 9(a). In the aqueous electrolyte, the SSGA-12 electrodes have a reasonable energy density of $8.5 \mathrm{Wh} \mathrm{kg}^{-1}$ at a power density of $625 \mathrm{~W} \mathrm{~kg}^{-1}$. As the energy density strongly 
depends on its operating voltage, the wide window of $0-3.5 \mathrm{~V}$ of the ionic liquid electrolyte made the SSGA-12 electrode show a much higher energy density: it exhibited a very high energy density of $80.0 \mathrm{Wh} \mathrm{kg}^{-1}$ at a power density of $438 \mathrm{~W} \mathrm{~kg}^{-1}$, which are about an order of magnitude higher than that of the previously reported 3D macroscopic graphene electrodes in aqueous electrolyte, and are even higher than some of the recently reported $\mathrm{N}$-doped graphene, activated carbon, or mesoporous carbon in ionic liquid electrolyte. ${ }^{40-43}$ In addition, even at a high power density of 9.1 $\mathrm{kW} \mathrm{kg}{ }^{-1}$, the SSGA-12 electrode retained a high energy density of $65.6 \mathrm{Wh} \mathrm{kg}^{-1}$, suggesting its excellent rate performance in the ionic liquid electrolyte. Fig. 9(b) shows the charge-discharge cycling performance of the SSGA-12 electrode in the aqueous electrolyte at the current density of $10 \mathrm{~A} \mathrm{~g}^{-1}$ and $80 \mathrm{~A} \mathrm{~g}^{-1}$, and in the ionic liquid electrolyte at the current density of $2 \mathrm{~A} \mathrm{~g}^{-1}$. After 2000 cycles, $97 \%$ of its initial capacitance was retained at the current density of $10 \mathrm{~A} \mathrm{~g}^{-1}$ in the aqueous electrolyte and $74 \%$ of its initial capacitance was retained even at $80 \mathrm{~A} \mathrm{~g} \mathrm{~g}^{-1}$. In ionic liquid electrolyte, the electrode can keep $94 \%$ of its initial capacitance at a current density of $2 \mathrm{~A} \mathrm{~g}^{-1}$ after 2000 cycles. These results indicate its good cycling stability in both electrolytes.

\section{Conclusions}

Single-walled carbon nanotube spaced graphene aerogels with desired porous structures were prepared for supercapacitor applications. Electrochemical tests showed that the graphene aerogel electrode delivered a high specific capacitance of 
$245.5 \mathrm{~F} \mathrm{~g}^{-1}$ in aqueous electrolyte $(5 \mathrm{M} \mathrm{KOH})$ and a high specific capacitance of 183.3 $\mathrm{F} \mathrm{g}^{-1}$ in ionic liquid electrolyte $\left(\mathrm{EMIMBF}_{4}\right)$. An extremely high energy density of 80.0 $\mathrm{Wh} \mathrm{kg}^{-1}$ in the ionic liquid electrolyte has been achieved with the graphene aerogel electrode. The graphene aerogel supercapacitor also showed excellent rate capability and long cycle life.

\section{Acknowledgements}

This work was supported by JST ALCA program, JSPS Grants-in-aid for Scientific Research (No. 22310074), and the Nanotechnology Network Project of the Ministry of MEXT, Japan.

\section{References}

[1] P. Simon, Y. Gogotsi, Nat Mater, 7 (2008) 845-854.

[2] J.R. Miller, P. Simon, Science, 321 (2008) 651-652.

[3] L.L. Zhang, X.S. Zhao, Chem. Soc. Rev, 38 (2009) 2520-2531.

[4] M.D. Stoller, S.J. Park, Y.W. Zhu, J.H. An, R.S. Ruoff, Nano Lett, 8 (2008) 3498-3502.

[5] J.Y. Luo, H.D. Jang, J.X. Huang, ACS Nano, 7 (2013) 1464-1471.

[6] Z. Wen, X. Wang, S. Mao, Z. Bo, H. Kim, S. Cui, G. Lu, X. Feng, J. Chen, Adv. Mater, 24 (2012) 5610-5616.

[7] Y. Zhu, S. Murali, M.D. Stoller, K.J. Ganesh, W. Cai, P.J. Ferreira, A. Pirkle, R.M. Wallace, K.A. Cychosz, M. Thommes, D. Su, E.A. Stach, R.S. Ruoff, Science, 332 (2011) 1537-1541.

[8] C. Liu, Z. Yu, D. Neff, A. Zhamu, B.Z. Jang, Nano Lett, 10 (2010) 4863-4868.

[9] F. Liu, S. Song, D. Xue, H. Zhang, Adv. Mater, 24 (2012) 1089-1094.

[10] Z. Niu, J. Chen, H.H. Hng, J. Ma, X. Chen, Adv. Mater, 24 (2012) 4144-4150.

[11] J.J. Yoo, K. Balakrishnan, J.S. Huang, V. Meunier, B.G. Sumpter, A. Srivastava, M. Conway, A.L.M. Reddy, J. Yu, R. Vajtai, P.M. Ajayan, Nano Lett, 11 (2011) 1423-1427.

[12] Y. Yoon, K. Lee, S. Kwon, S. Seo, H. Yoo, S. Kim, Y. Shin, Y. Park, D. Kim, Y.-y. Choi, H. Lee, ACS Nano, 8 (2014) 4580-4590

[13] J. Lee, S. Kim, J. Yoo, J. Jang, ACS nano, 7 (2013) 6047-6055.

[14] Y.X. Xu, K.X. Sheng, C. Li, G.Q. Shi, ACS Nano, 4 (2010) 4324-4330.

[15] Y. Zhao, C. Hu, Y. Hu, H. Cheng, G. Shi, L. Qu, Angew. Chem. Int. Ed, 48 (2009) 5864-5868. 
[16] Z.S. Wu, A. Winter, L. Chen, Y. Sun, A. Turchanin, X. Feng, K. Mullen, Adv. Mater, 24 (2012) 5130-5135.

[17] S. Ye, J. Feng, ACS App. Mater. Interfaces, 6 (2014) 9671-9679.

[18] H.F. Ju, W.L. Song, L.Z. Fan, J. Mater. Chem. A, 2 (2014) 10895.

[19] Q. Cheng, J. Tang, J. Ma, H. Zhang, N. Shinya, L.C. Qin, Phys. Chem. Chem. Phys., 13 (2011) 17615-17624.

[20] F. Zhang, J. Tang, N. Shinya, L.C. Qin, Chem. Phys. Lett, 584 (2013) 124-129.

[21] J. Yan, T. Wei, B. Shao, F. Ma, Z. Fan, M. Zhang, C. Zheng, Y. Shang, W. Qian, F. Wei, Carbon, 48 (2010) 1731-1737.

[22] X. Du, H. Song, X. Chen, J. Mater. Chem., 22 (2012) 13091.

[23] Y. Yu, Y. Sun, C. Cao, S. Yang, H. Liu, P. Li, P. Huang, W. Song, J. Mater. Chem. A, 2 (2014) 7706.

[24] Y. Zhu, L. Li, C. Zhang, G. Casillas, Z. Sun, Z. Yan, G. Ruan, Z. Peng, A.R. Raji, C. Kittrell, R.H. Hauge, J.M. Tour, Nat. Commun, 3 (2012) 1225.

[25] F. Zhang, J. Tang, Z. Wang, L.C. Qin, Chem. Phys. Lett, 590 (2013) 121-125.

[26] P. Chen, J.J. Yang, S.S. Li, Z. Wang, T.Y. Xiao, Y.H. Qian, S.H. Yu, Nano Energy, 2 (2013) 249-256.

[27] Z.L. Yuxi Xu, Xiaoqing Huang, Yuan Liu, Yu Huang, Xiangfeng Duan, ACS nano, 7 (2013) 4042-4049.

[28] W. Chen, L. Yan, Nanoscale, 3 (2011) 3132-3137.

[29] Z.S. Wu, A. Winter, L. Chen, Y. Sun, A. Turchanin, X. Feng, K. Mullen, Adv. Mater, 24 (2012) 5130-5135.

[30] Y. Zhao, C. Hu, L. Song, L. Wang, G. Shi, L. Dai, L. Qu, Energ Environ Sci, 7 (2014) 1913-1918.

[31] L. Qiu, X. Yang, X. Gou, W. Yang, Z.F. Ma, G.G. Wallace, D. Li, Chem. Eur. J, 16 (2010) 10653-10658.

[32] L. Tian, M.J. Meziani, F. Lu, C.Y. Kong, L. Cao, T.J. Thorne, Y.P. Sun, ACS App. Mater. Interfaces, 2 (2010) 3217-3222.

[33] L. Borchardt, M. Oschatz, S. Kaskel, Mater. Horiz, 1 (2014) 157.

[34] Z. Sui, Q. Meng, J. Li, J. Zhu, Y. Cui, B. Han, J. Mater. Chem. A, 2 (2014). 9891-9898

[35] Z.J. Jiang, Z. Jiang, W. Chen, J. Power Sources, 251 (2014) 55-65.

[36] L. Zhang, F. Zhang, X. Yang, G. Long, Y. Wu, T. Zhang, K. Leng, Y. Huang, Y. Ma, A. Yu, Y. Chen, Sci Rep, 3 (2013) 1408-1416

[37] C.L. Liu, W. Dong, G. Cao, J. Song, L. Liu, Y. Yang, J. Electrochem. Soc, 155 (2008) F1-F7.

[38] F. Xu, R. Cai, Q. Zeng, C. Zou, D. Wu, F. Li, X. Lu, Y. Liang, R. Fu, J. Mater. Chem, 21 (2011) 1970.

[39] H. Zhong, F. Xu, Z. Li, R. Fu, D. Wu, Nanoscale, 5 (2013) 4678-4682.

[40] M. Sathish, S. Mitani, T. Tomai, I. Honma, J. Mater. Chem. A, 2 (2014) 4731-4738.

[41] M. Galinski, K. Babel, K. Jurewicz, J. Power Sources, 228 (2013) 83-88.

[42] A. Vu, X.Y. Li, J. Phillips, A.J. Han, W.H. Smyrl, P. Buhlmann, A. Stein, Chem Mater, 25 (2013) 4137-4148.

[43] Z. Lei, Z. Liu, H. Wang, X. Sun, L. Lu, X.S. Zhao, J. Mater. Chem. A, 1 (2013) 2313-2321. 


\section{Figure Captions}

Table 1. Specific surface area and pore volume of graphene aerogels (GAs) and single-walled carbon nanotube spaced graphene aerogels (SSGAs).

Table 2. Comparison of ESR and $\mathrm{R}_{\mathrm{ct}}$ of various graphene electrodes in aqueous and ionic liquid electrolyte obtained from the Nyquist plot of the EIS data.

Figure 1. (a) Schematic illustration and (b) photographs of the fabrication of homogeneous SSGAs with maco-pores and integrated 3D structure. The resulting SSGA is ultra-light in weight.

Figure 2. (a-b) SEM images and (c-d) TEM images of as-prepared SSGAs. Carbon nanotubes are distributed in graphene structure homogenously.

Figure 3. (a) XRD patterns of GO, GAs, and SSGAs; (b) FT-IR spectra of GO, GAs, and SSGAs; and (c) Raman spectra of SWCNT, GAs, and SSGAs with various concentration of SWCNTs.

Figure 4. $\mathrm{N}_{2}$ adsorption/desorption analysis of GA, SSGAs and SWCNTs. (a) $\mathrm{N}_{2}$ isotherm curves at $77.4 \mathrm{~K}$. (b) Plots of cumulative pore volume versus pore diameter obtained from the adsorption isotherms.

Figure 5. Electric double-layer capacitor performance in aqueous electrolyte. (a-d) CVs of the GA, SSGA-16, SSGA-12, and SSGA-8 at different scan rates from 50 to $500 \mathrm{mV} \mathrm{s}^{-1}$. (e) CVs of different samples at the scan rate of $100 \mathrm{mV} \mathrm{s}^{-1}$. (f) 
Dependence of specific capacitance on scan rate of the four graphene aerogel electrodes. The SSGA-12 sample exhibited the best electrochemical performance.

Figure 6. Electric double-layer capacitor performance of the graphene agrogel electrodes in aqueous electrolyte. (a-d) Galvanostatic charge/discharge curves of GA, SSGA-16, SSGA-12, and SSGA-8 at different current densities from 5 to $80 \mathrm{~A}$ $\mathrm{g}^{-1}$. The current density was calculated based on the mass of a single electrode. (e) Charge/discharge curves of different samples at current densities of $5 \mathrm{~A} \mathrm{~g}^{-1}$. (f) Dependences of specific capacitance on discharge current density for the four aerogel electrodes.

Figure 7. Electric double-layer capacitor performance in ionic liquid electrolyte. (a) CVs of GAs, SSGA-16, SSGA-12, and SSGA-8 at scan rate of $50 \mathrm{mV} \mathrm{s}^{-1}$. (b) CVs of SSGA-12 at different scan rates from 20 to $100 \mathrm{mV} \mathrm{s}^{-1}$. (c-d) Galvanostatic charge/discharge curves of different samples at current density of 1 and $5 \mathrm{~A} \mathrm{~g} \mathrm{~g}^{-1}$, respectively. (e) Charge/discharge curves of SSGA-12 at different current densities from 1 to $10 \mathrm{~A} \mathrm{~g}^{-1}$. (f) Dependences of specific capacitance on discharge current density for the four aerogel electrodes.

Figure 8. Nyquist plots of GA, SSGA-16, SSGA-12, and SSGA-8 supercapacitor electrode in (a) aqueous electrolyte and (b) ionic liquid electrolyte.

Figure 9. (a) Ragone plot and (b) Cycling performance of the SSGA-12 electrode in aqueous and ionic liquid electrolyte. 
Table 1 Specific surface area and pore volume of graphene aerogel (GA) and single-walled carbon nanotube spaced graphene aerogels (SSGAs), and single-walled carbon nanotubes (SWNTs).

\begin{tabular}{cccc}
\hline Sample & $\begin{array}{c}\text { Specific Surface Area } \\
\left(\mathrm{m}^{2} \mathrm{~g}^{-1}\right)\end{array}$ & $\begin{array}{c}\text { Pore Volume of Pores Smaller } \\
\text { Than 20 } \mathrm{nm}\left(\mathrm{cc} \mathrm{g}^{-1}\right)\end{array}$ & $\begin{array}{c}\text { Ratio of Pore Volume of } \\
\text { Pores Smaller Than 20nm }\end{array}$ \\
\hline GA & 430 & 0.22 & $44.0 \%$ \\
SSGA-16 & 551 & 0.38 & $89.2 \%$ \\
SSGA-12 & 807 & 0.47 & $88.2 \%$ \\
SSGA-8 & 712 & 0.43 & $87.6 \%$ \\
SWCNTs & 344 & 0.26 & $70.7 \%$ \\
\hline
\end{tabular}


Table 2 Comparison of ESR and $\mathrm{R}_{\mathrm{ct}}$ of various graphene electrodes in aqueous and ionic liquid electrolyte obtained from the Nyquist plot of the EIS data.

\begin{tabular}{ccccc}
\hline & \multicolumn{2}{c}{ Aqueous Electrolyte } & \multicolumn{2}{c}{ Ionic Liquid Electrolyte } \\
\hline Electrode & $\mathrm{ESR}(\mathrm{m} \Omega)$ & $\mathrm{R}_{\mathrm{ct}}(\mathrm{m} \Omega)$ & $\mathrm{ESR}(\mathrm{m} \Omega)$ & $\mathrm{R}_{\mathrm{ct}}(\mathrm{m} \Omega)$ \\
GAs & 166.6 & 121.4 & 12.0 & 18.5 \\
SSGA-16 & 141.2 & 67.4 & 12.8 & 11.5 \\
SSGA-12 & 132.2 & 56.5 & 11.7 & 5.0 \\
SSGA-8 & 129.0 & 52.3 & 11.5 & 4.3 \\
\hline
\end{tabular}


(a)

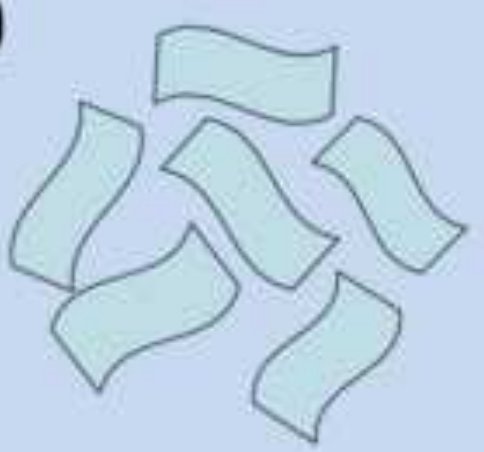

GO

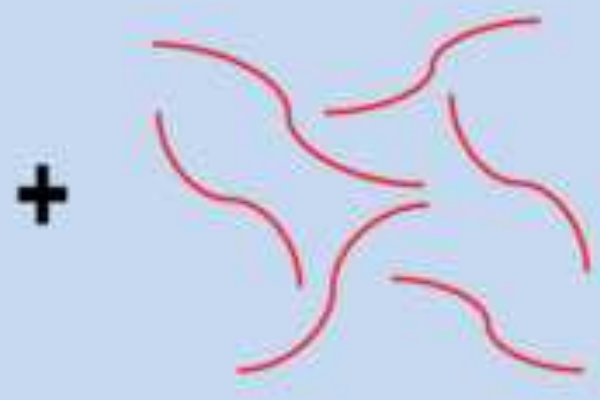

SWCNT

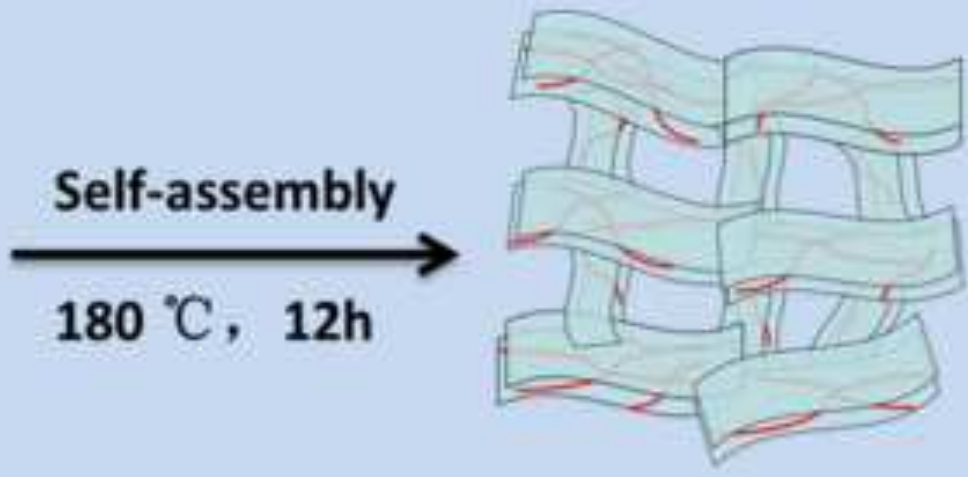

3D SWCNT spaced Graphene aerogels

(b)

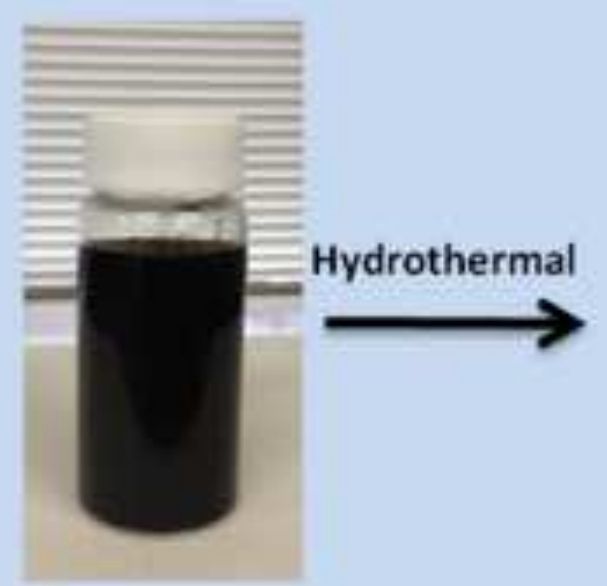

Mixed GO and SWCNT
SWCNT spaced Graphene Hydrogels
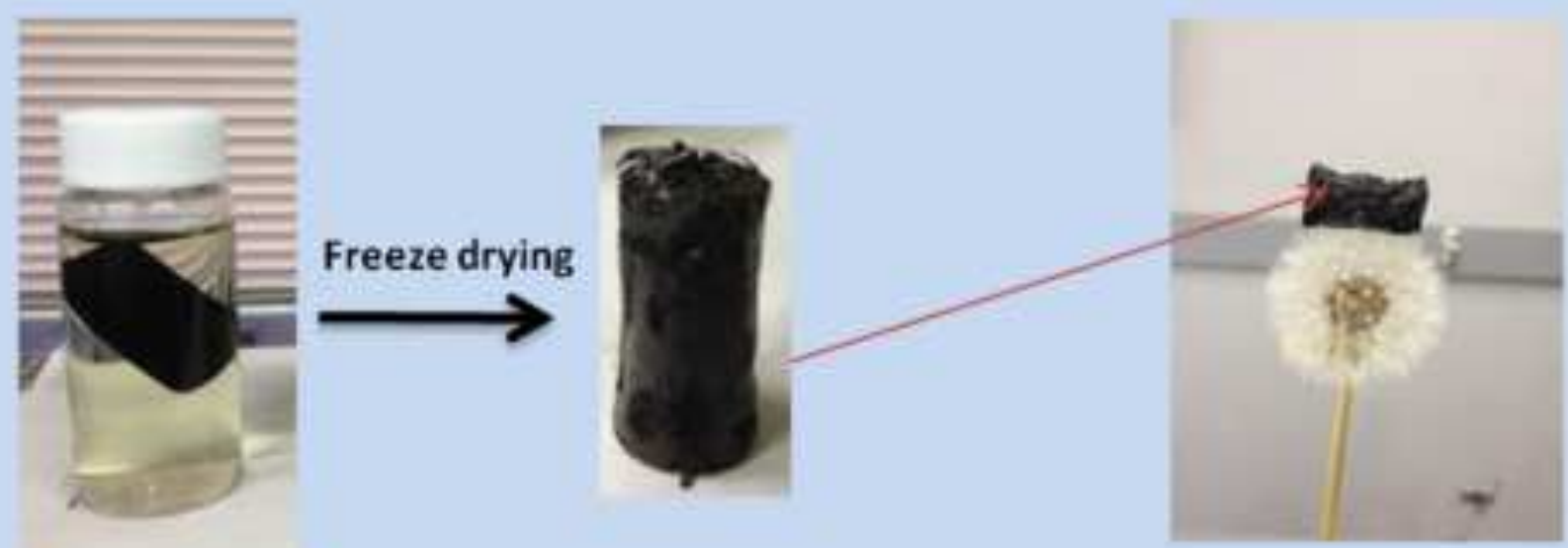

SWCNT spaced Graphene Aerogels
Ultralight composite aerogels 

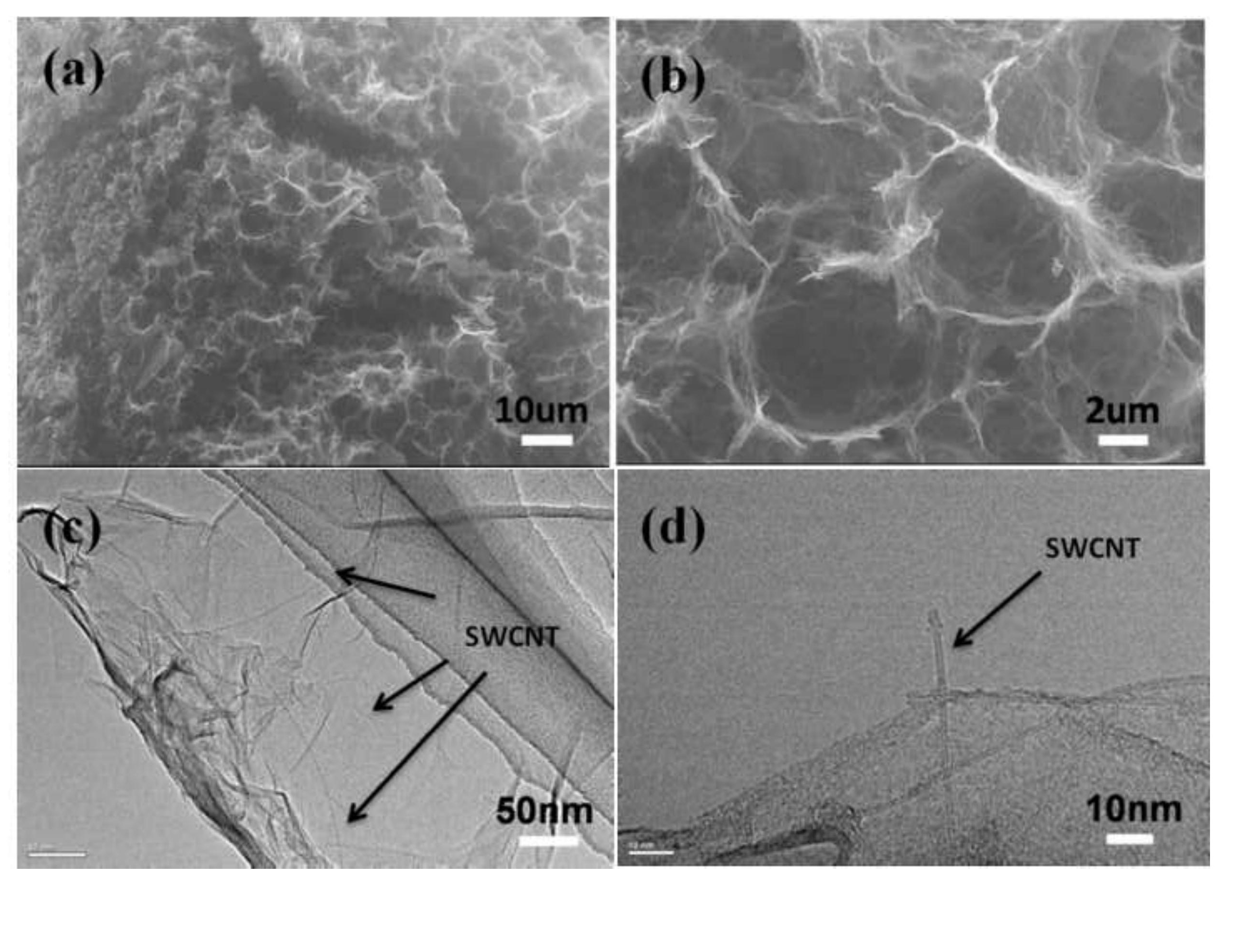
(9)

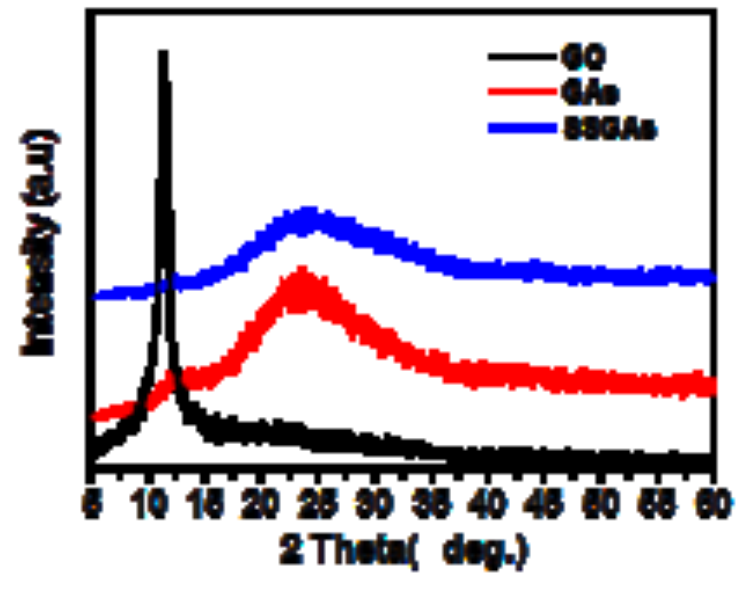

(b)

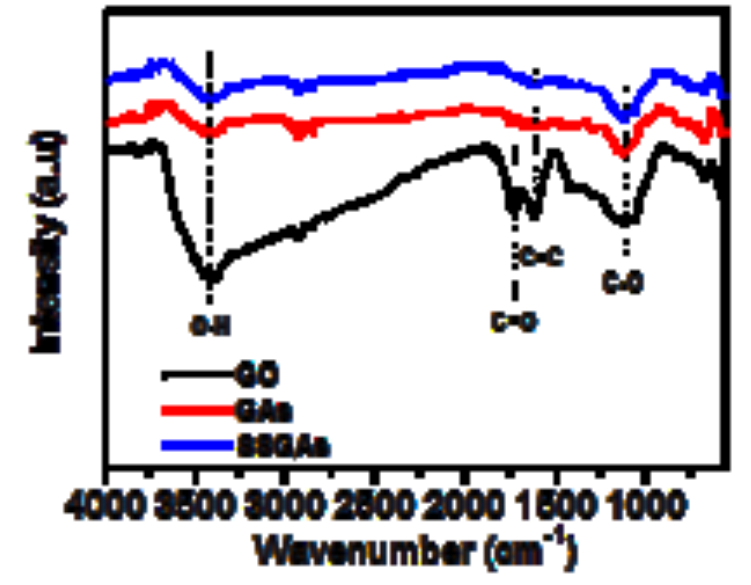

(c)

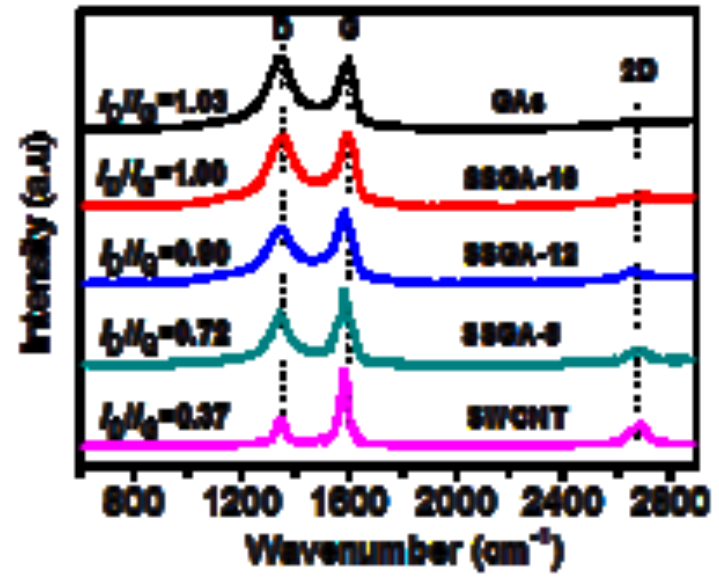


Figure 4

(a)
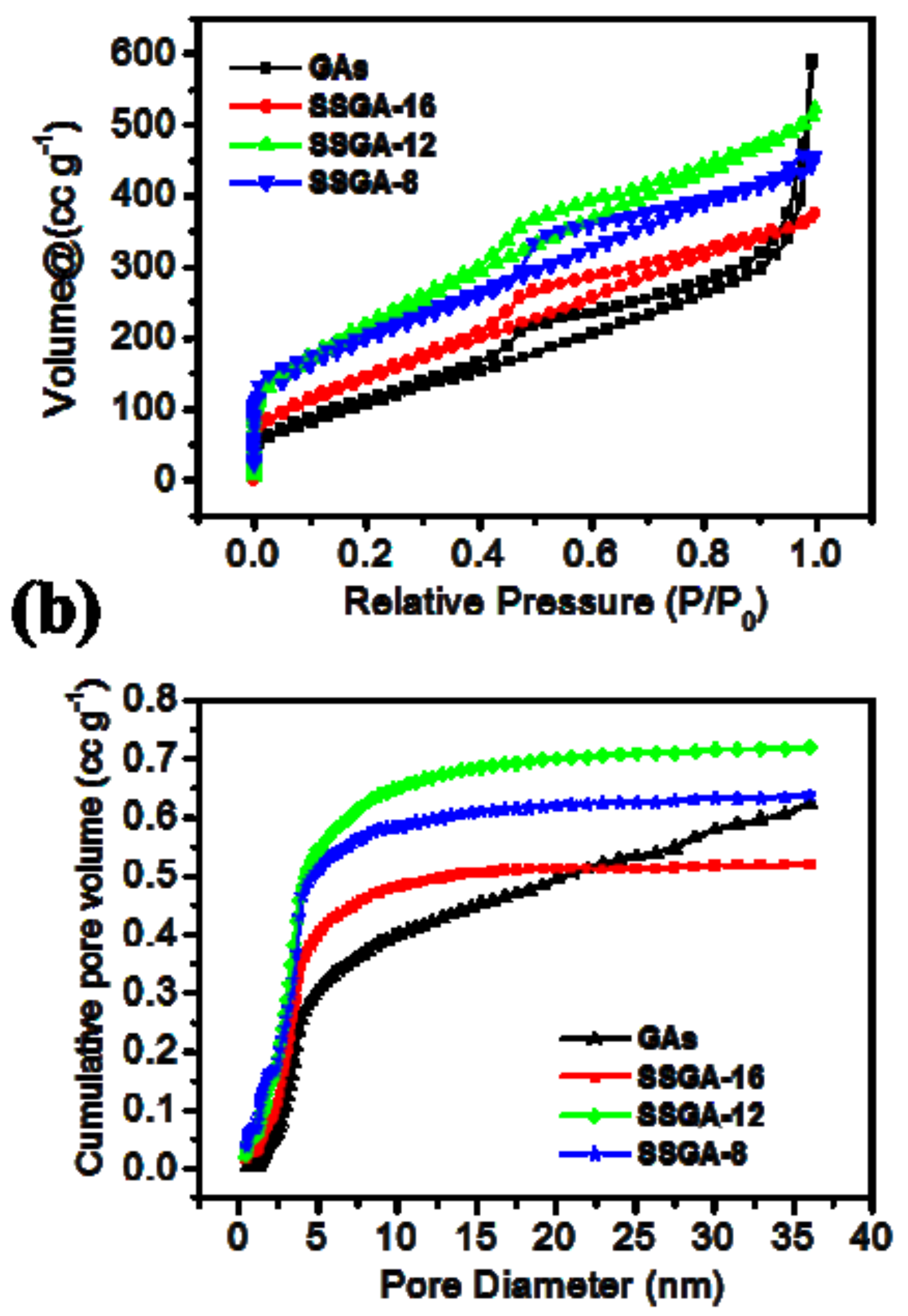
(a)

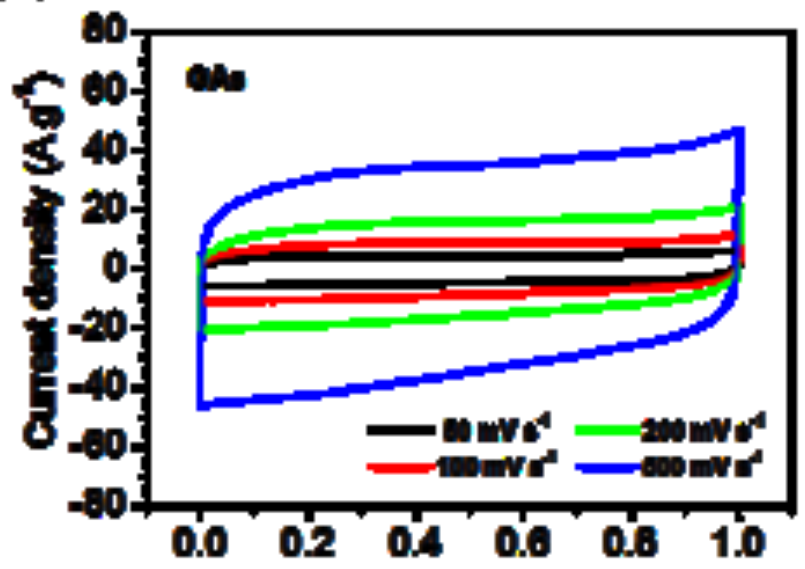

(c) Polwintal(V)

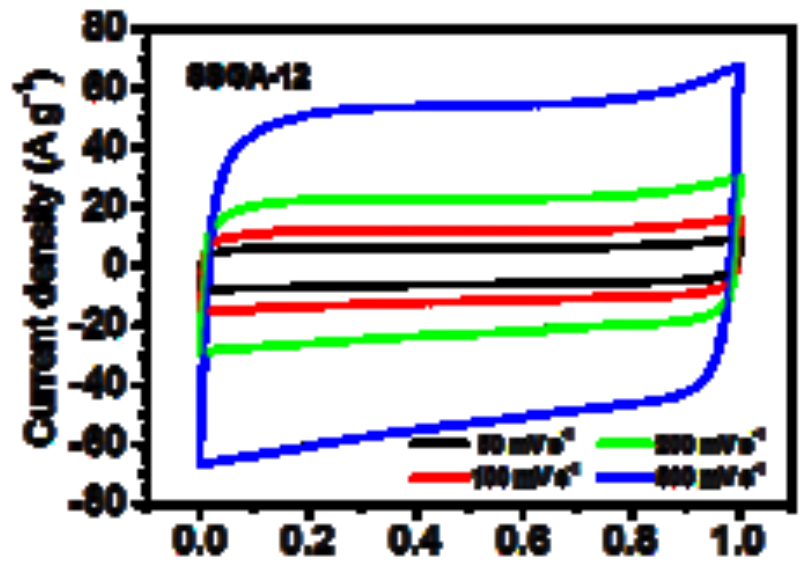

(e)

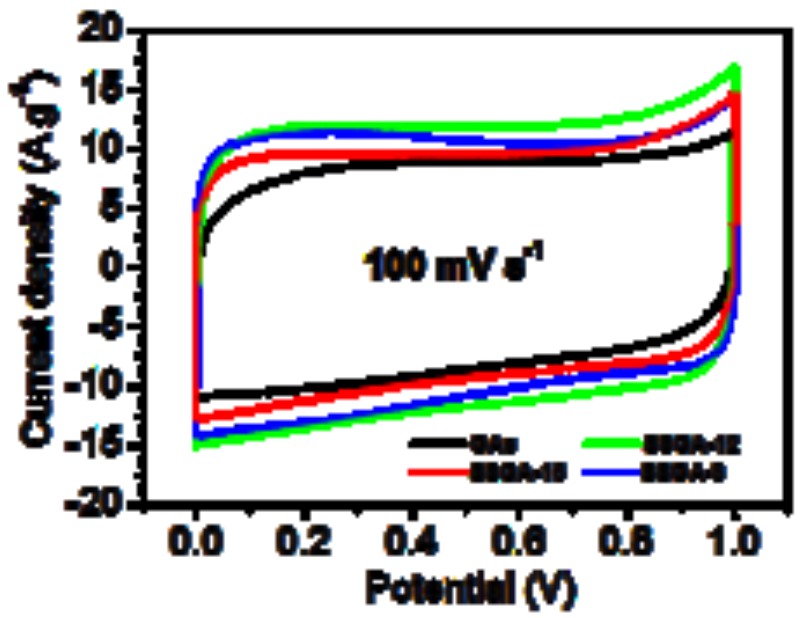

(b)

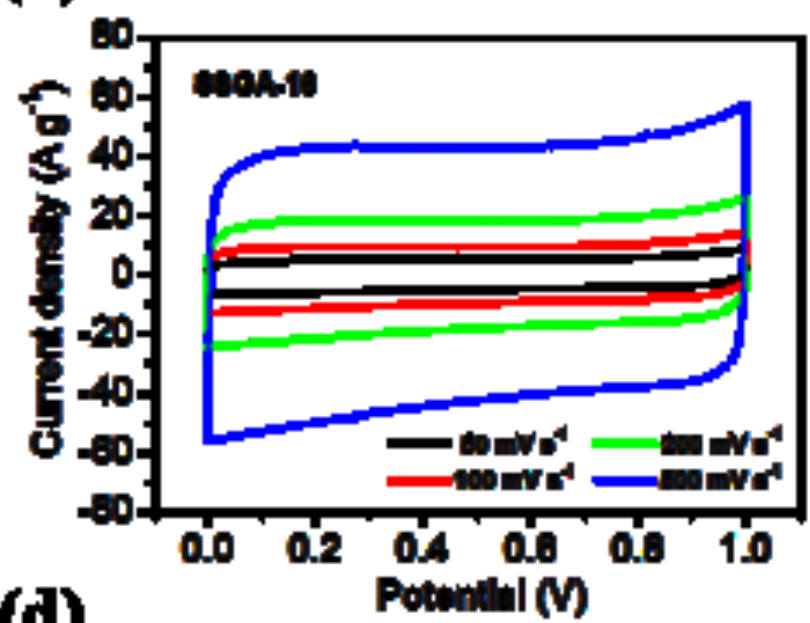

(d)

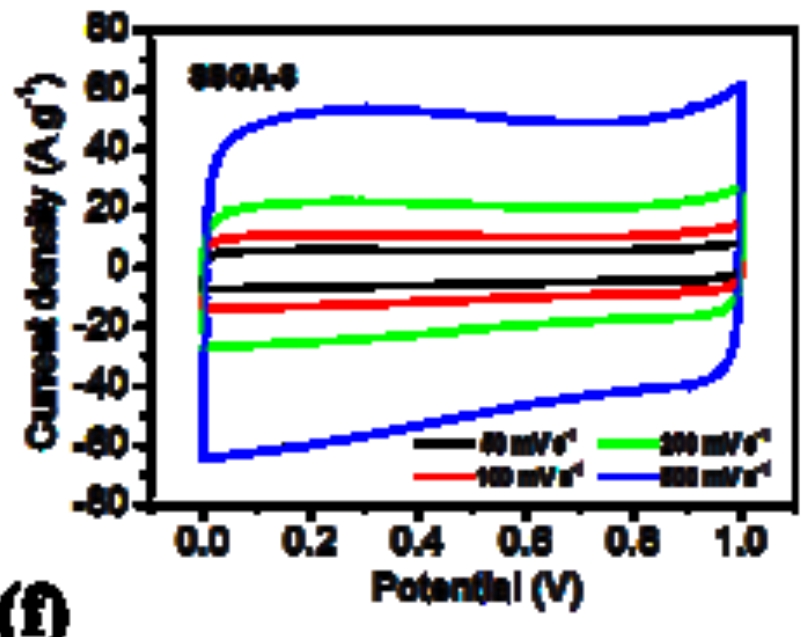

(f)

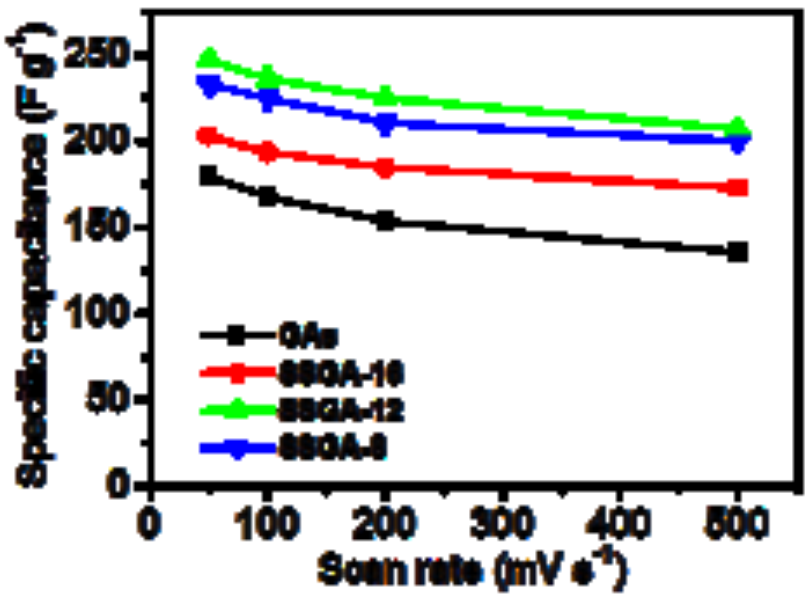


(a)

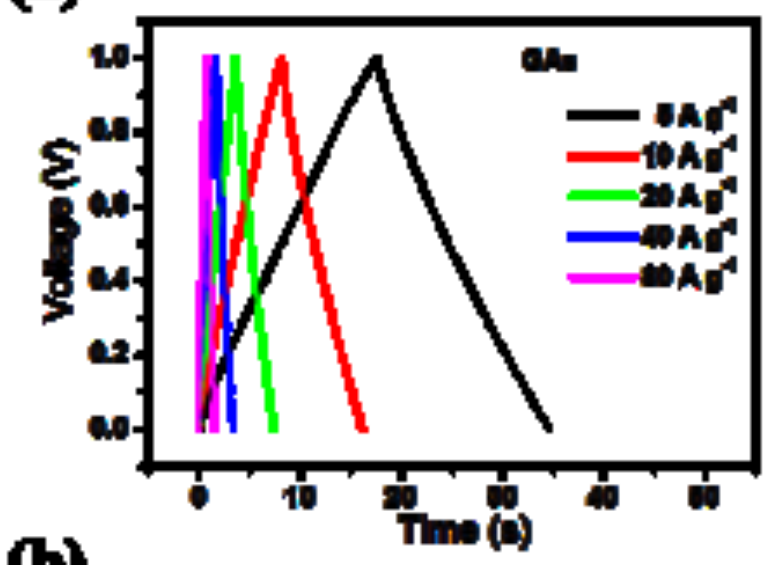

(b)

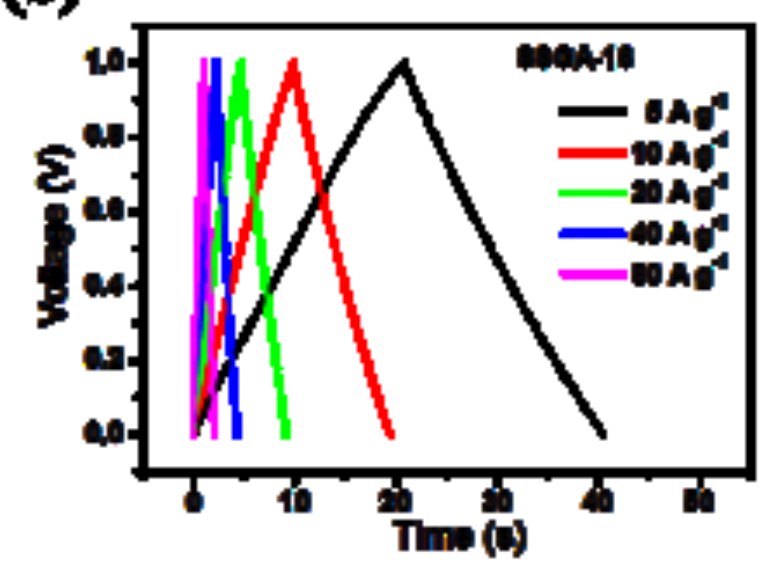

(c)

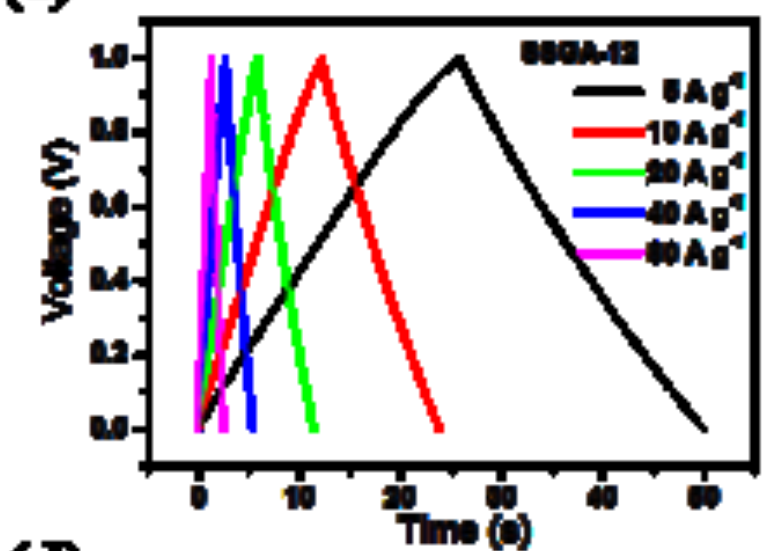

(d)

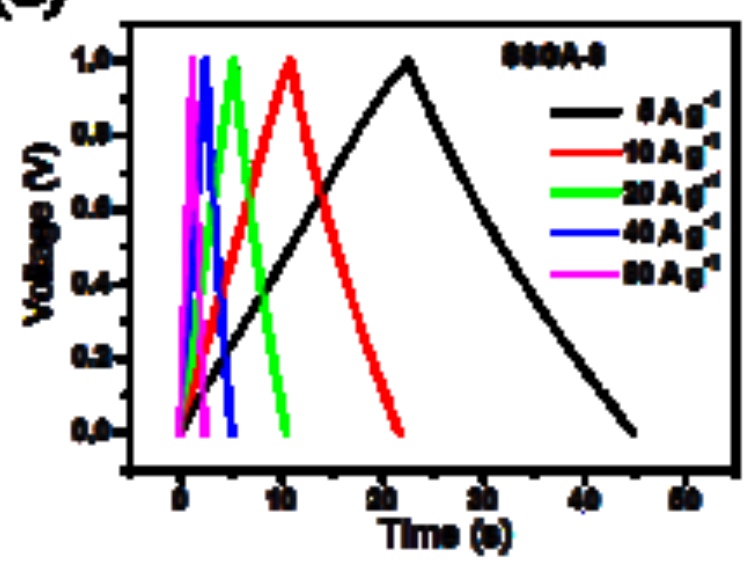

(e)

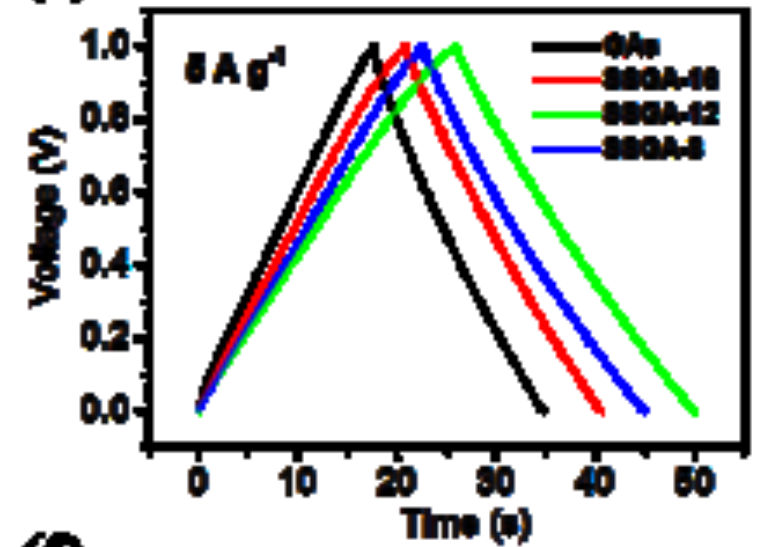

(D)

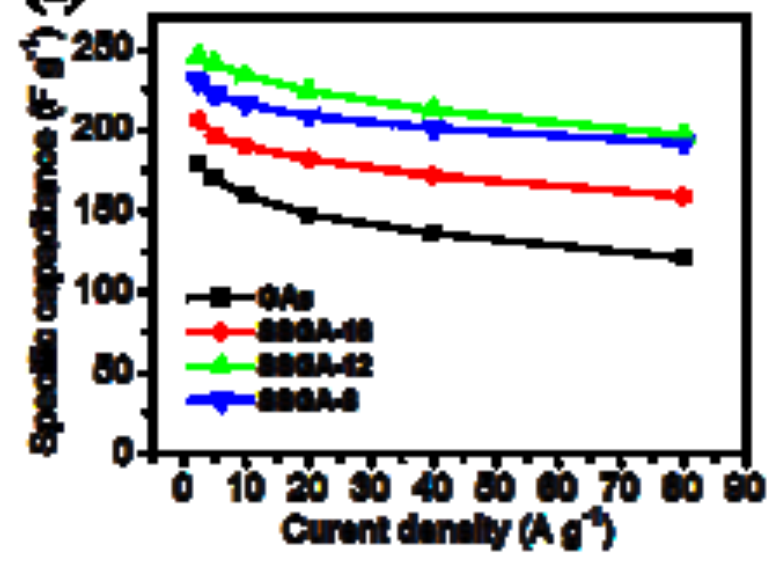


(a)

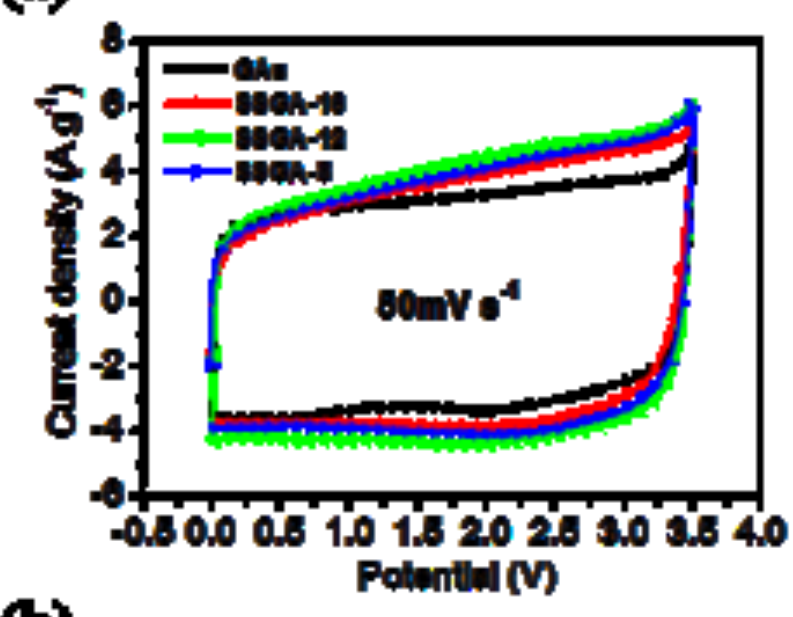

(b)

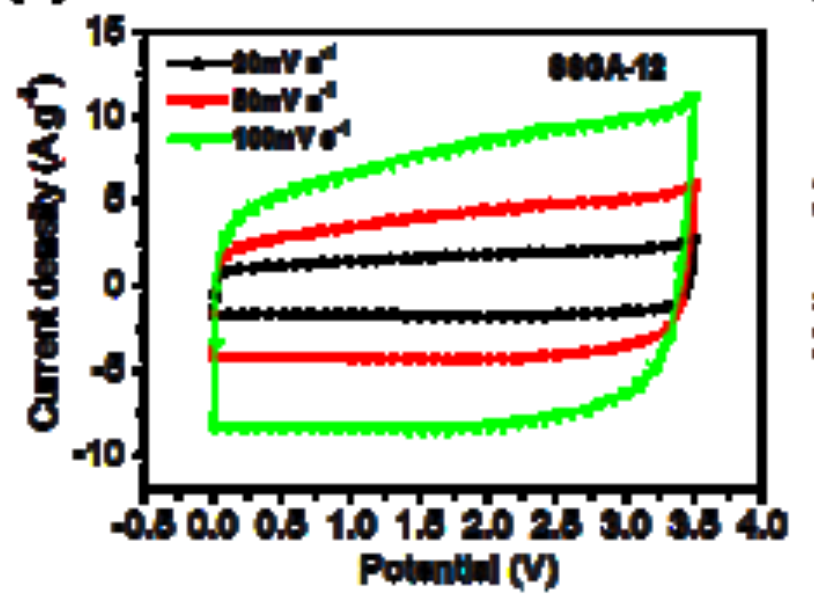

(c)

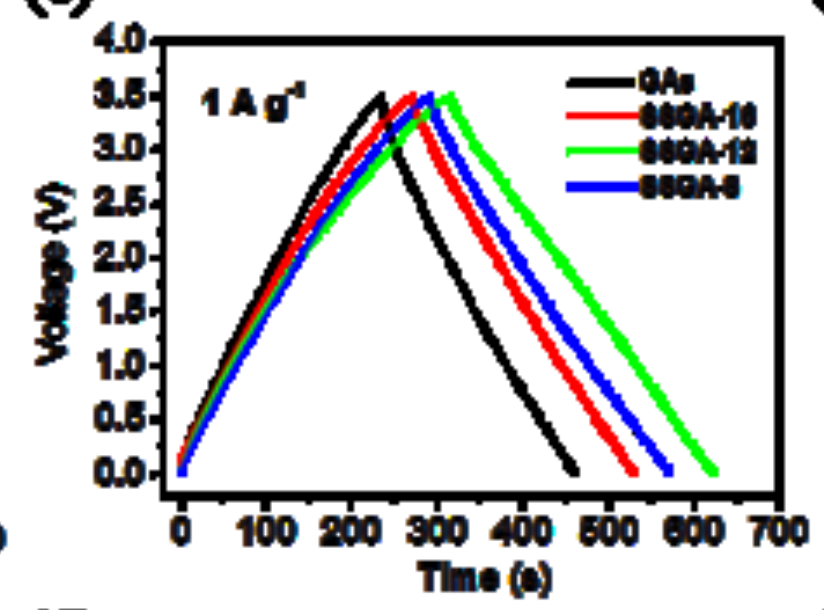

(d)

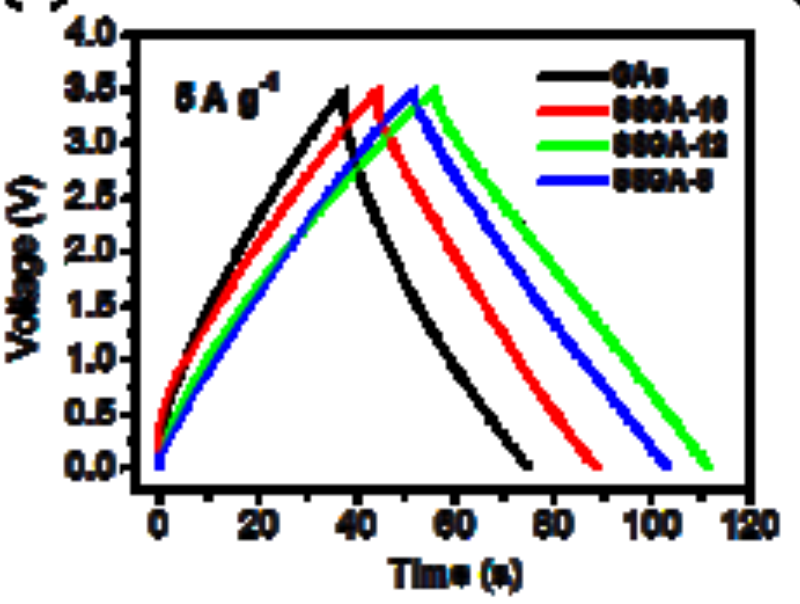

(e)

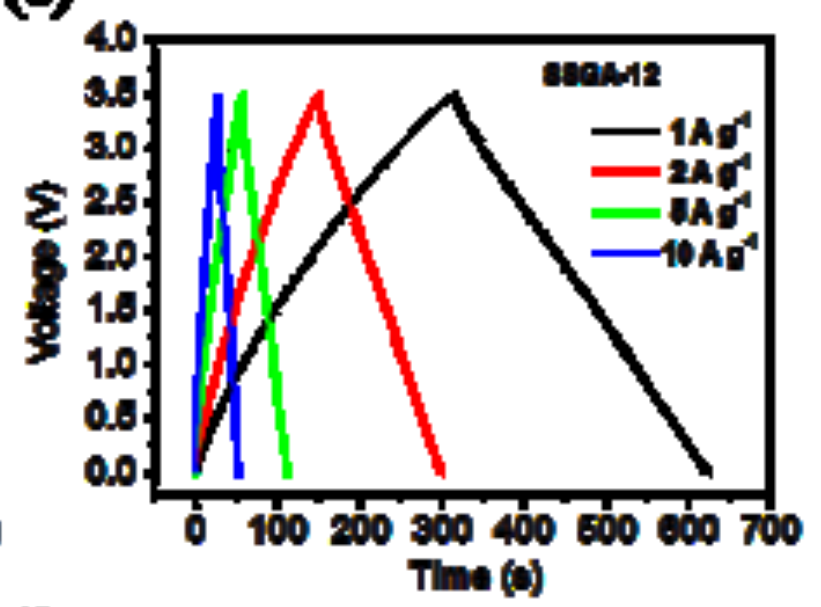

(i)

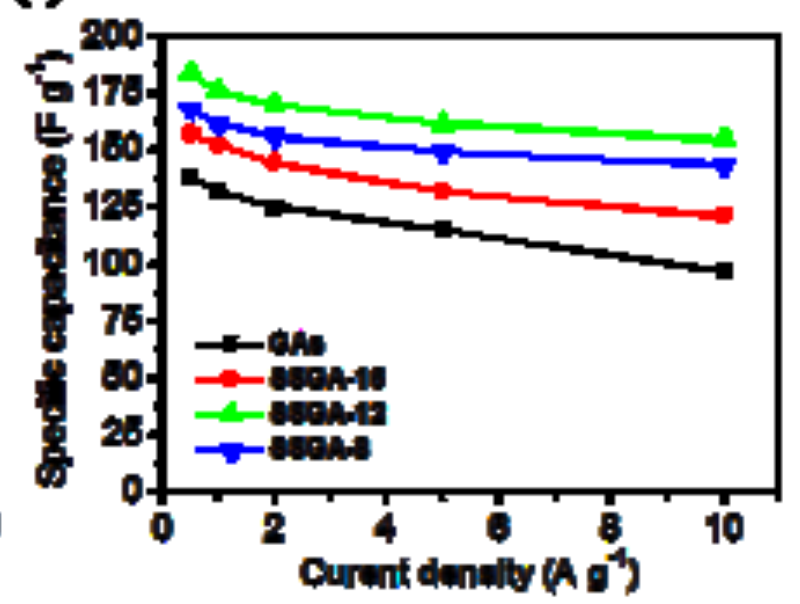


(a)

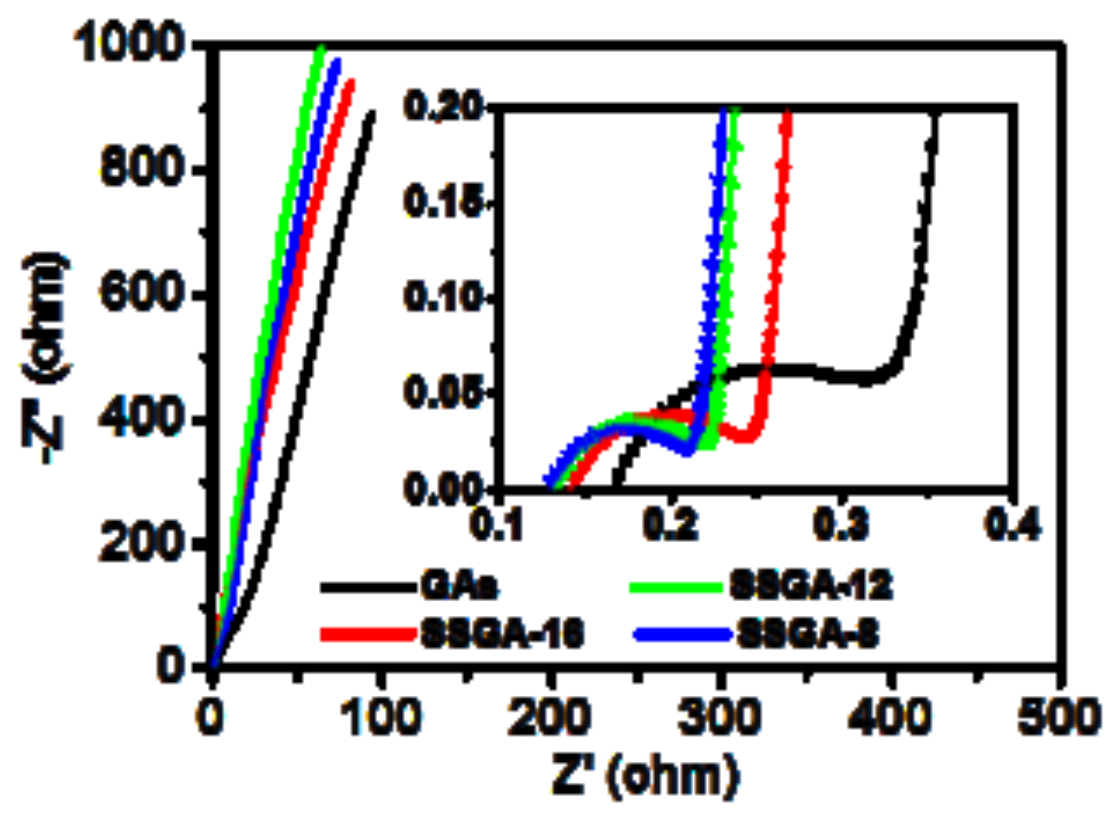

(b)

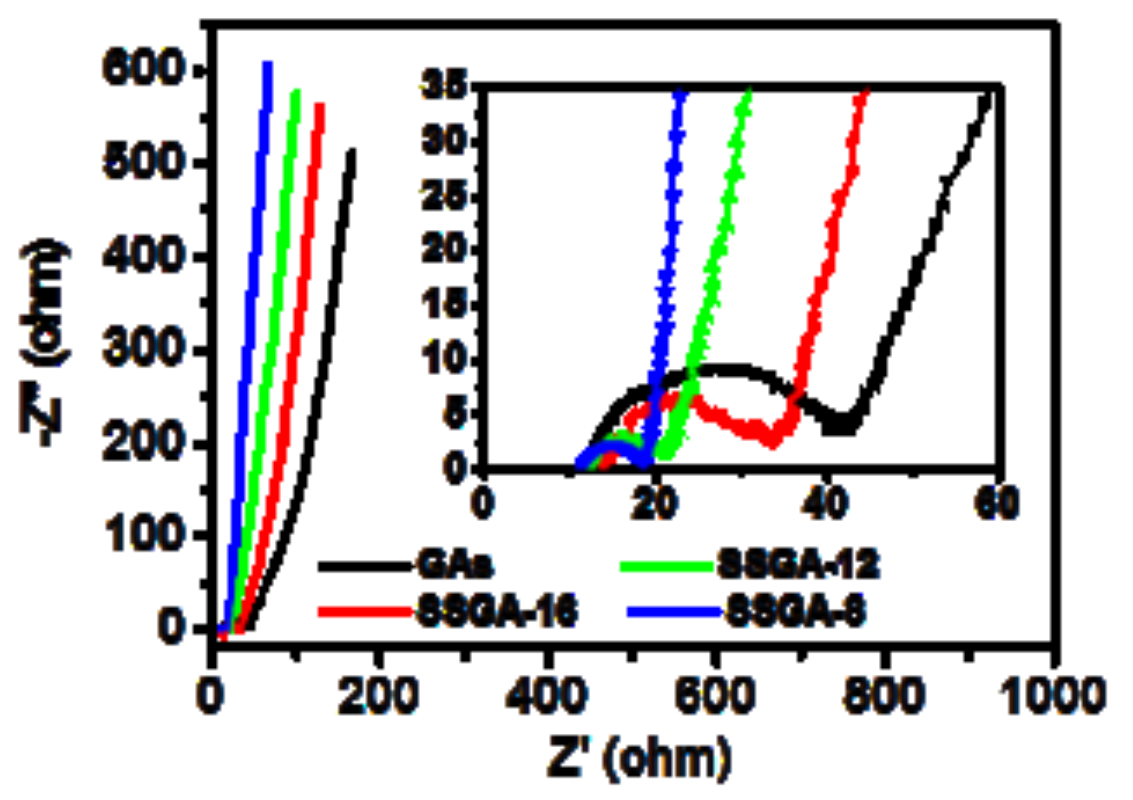


(a)

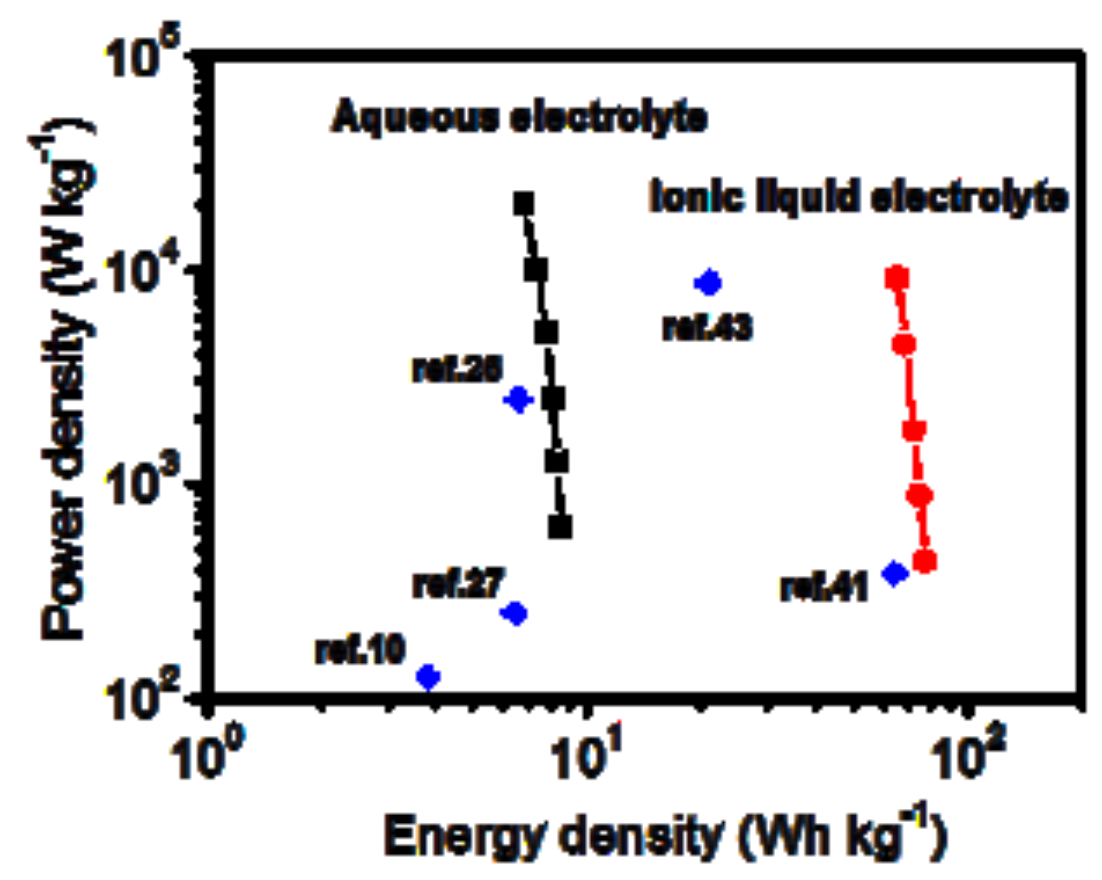

(b)

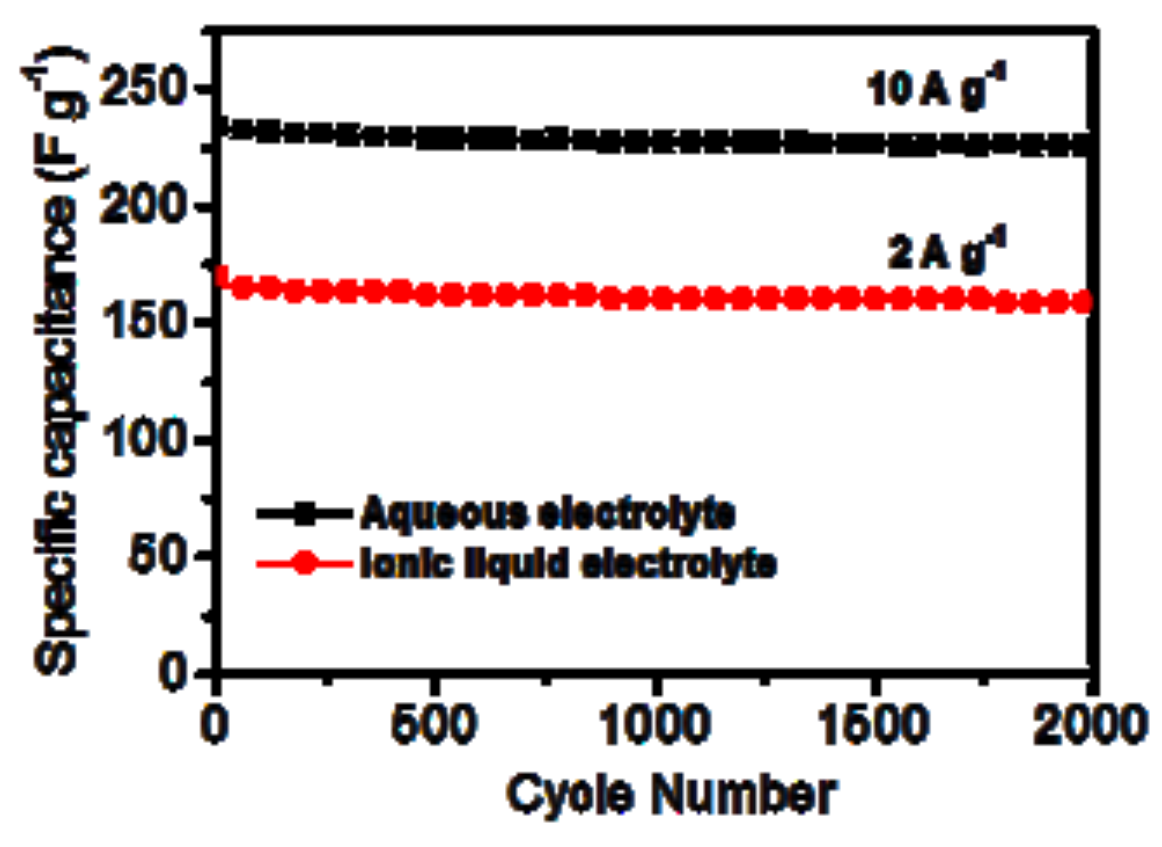



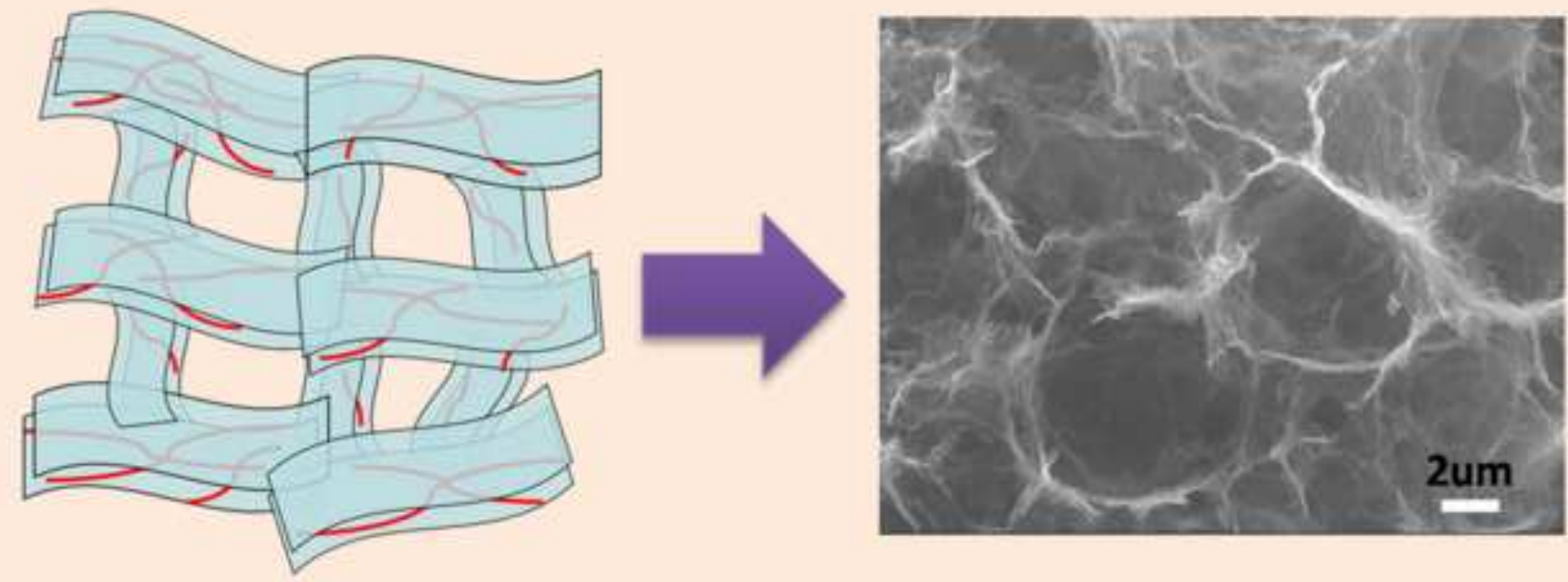

SWCNT spaced Graphene aerogels for high performance supercapacitors
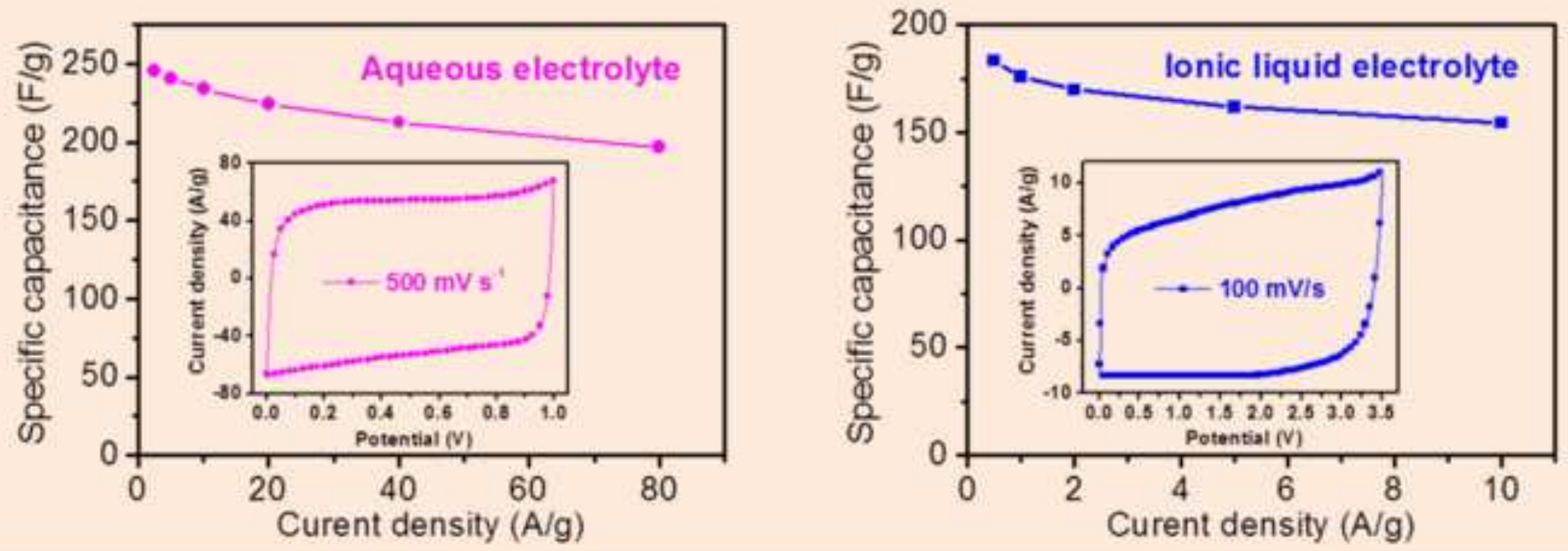